\title{
An Artificial Intelligence-Based Smart System for Early Glaucoma Recognition Using OCT Images
}

Law Kumar Singh, Department of Computer Science and Engineering, School of Engineering and Technology, Sharda University, Knowledge Park III, Greater Noida, India \& Department of Computer Science and Engineering, Hindustan College of Science and Technology, Mathura, India

iD https://orcid.org/0000-0002-7073-6852

Pooja, Department of Computer Science and Engineering, School of Engineering and Technology, Sharda University, Knowledge Park III, Greater Noida, India

Hitendra Garg, Department of Computer Engineering and Applications, GLA University, Mathura, India

Munish Khanna, Department of Computer Science and Engineering, Hindustan College of Science and Technology, Mathura, India

\begin{abstract}
Glaucoma is a progressive and constant eye disease that leads to a deficiency of peripheral vision and, at last, leads to irrevocable loss of vision. Detection and identification of glaucoma are essential for earlier treatment and to reduce vision loss. This motivates us to present a study on intelligent diagnosis system based on machine learning algorithm(s) for glaucoma identification using three-dimensional optical coherence tomography (OCT) data. This experimental work is attempted on 70 glaucomatous and 70 healthy eyes from combination of public (Mendeley) dataset and private dataset. Forty-five vital features were extracted using two approaches from the OCT images. K-nearest neighbor (KNN), linear discriminant analysis (LDA), decision tree, random forest, support vector machine (SVM) were applied for the categorization of OCT images among the glaucomatous and non-glaucomatous class. The largest AUC is achieved by KNN (0.97). The accuracy is obtained on fivefold cross-validation techniques. This study will facilitate to reach high standards in glaucoma diagnosis.
\end{abstract}

\section{KEYWORDS}

Cup Diameter, Cup-to-Disc Ratio (CDR), Decision Tree, Disc Diameter, Glaucomatous, Internal Limiting Membrane (ILM) Thickness, KNN, LDA, OCT, Random Forest, Retinal Pigmented Epithelium (RPE), SVM

\section{INTRODUCTION}

Glaucoma is the second most crucial optic 'eye' disease in this world. As per sources in 2010, approximately 60 million populations cross-sectional to be disease infected, and this count is increasing above 20 million in the period 2020. It does no repairable damage to the central part of the optic nerves that can lead to making the person blind. Hence, sensing Glaucoma during the initial stage is very imperative. Generally, doctors focus on the area of the optic disc \& optic cup and find the edges though optic nerve examination. They assure the glaucoma presence they identify the increased size of the optic cup. One of the essential features is to identify the ratio of the height of the Cup to the 
disc; this is the crucial indicator for identifying Glaucoma. Among the patients, if the Cup-to-Disc ratio (CDR) value is at least 0.5 , it may be considered as the glaucomatous eye.

Human eye mainly has three layers. The outer layer: Sclera, which is used to protect the eyeball; Second layer: Choroid and the innermost layer: Retina."Retina" is liable in vision because of the presence of photoreceptors. Researchers have invented many techniques which are used for detecting retinal disorder. These techniques include fundus photography, fluorescein angiography, and the OCT.

Figure 1. OCT image with macular edema

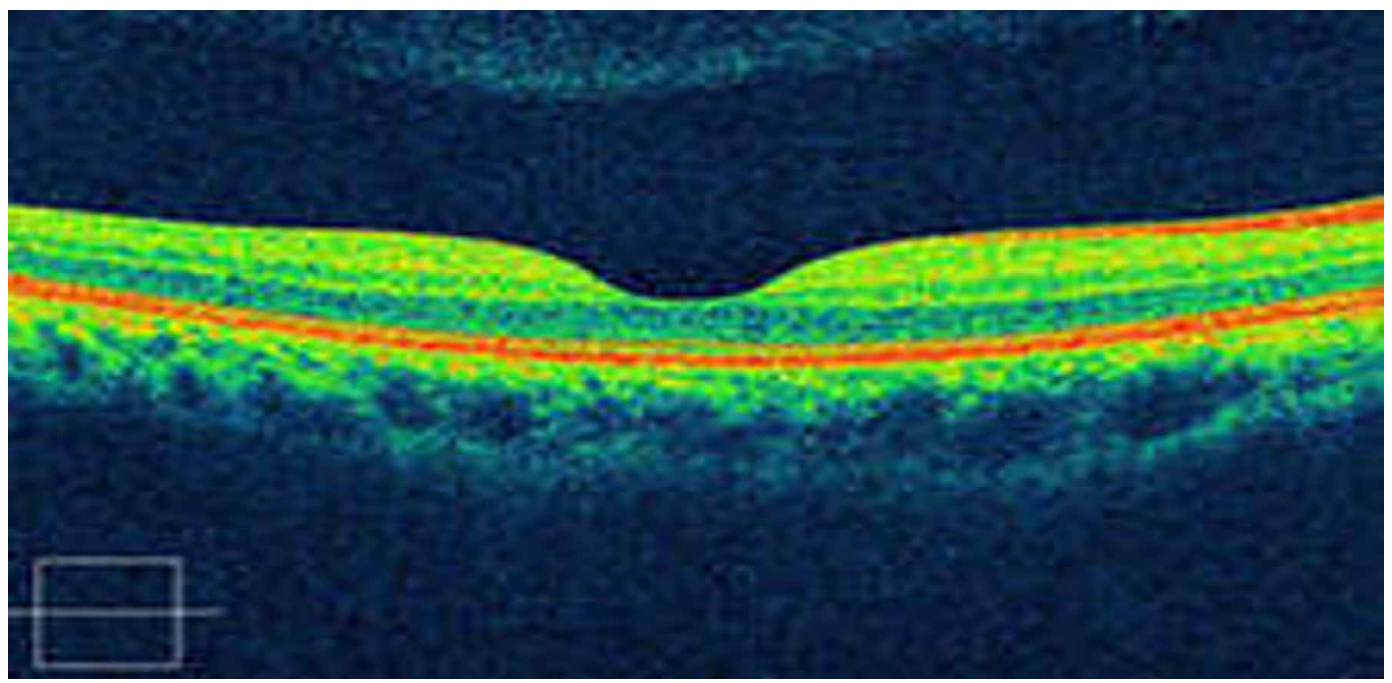

\subsection{Optical Coherence Tomography}

OCT stands for optical coherence tomography, which is having the property that it is a noninvasive diagnostic technique used for manufacturing or producing the view of the retina in a cross-sectional way (Burgansky-Eliash et al., 2005).OCT imaging technology uses OCT cameras, which have low coherence interferometers in which the low coherence visible light is always allowed to penetrate the entire human retina and it reflects interferometer producing a cross-sectional image of the retina. Low coherence of lightning is used for producing images of resolutions - the basic principles of the OCT imaging technique on the (Michelson type interferometer). A considerable benefit of the OCT imaging system (Fig. 1) is always that it can detect retinal disorders earlier than other techniques.

It has been observed that the majority of the work, to date, accomplished by the researcher's fraternity is performed on fundus images for glaucoma detection. However, we have used OCT images as there are multiple advantages of OCT images over fundus images. OCT has higher sensitivity than the fundus image for the detection of early Glaucoma. It is a non-invasive technology and has a higher ability to detect small changes in the subretinal layer than the fundus image. This image picks up the earliest signs of diseases. OCT evaluates disorders of the optic nerve and changes to the fibers of the optic nerve. It detects changes caused by Glaucoma.

Moreover OCT(Khalil et al., 2014) images also have higher resolution.OCT provides topographical information about retinal ganglion cells and Retinal Nerve Fiber Layer (RNFL) abnormalities.OCT images allow for direct visual analysis of the scans, much the way MRI scans are analyzed, rather than depending entirely upon computer-driven summary statistics. It can produce real-time a crosssectional image of an object, i.e. a two-dimensional image in the space.OCT images give more accuracy 
as compared to the fundus images. Analysis of OCT is done by the degradation of a separate layer, which increases the efficiency of an image.OCT provides detailed structural information on clinical abnormalities. This method has been extensively applied to the setting of ophthalmology; this method is popular due to its ability to perform high-resolution cross-sectional imaging and diagnosing the changes in the structure of the eye during the progression of this disease.

\subsection{OAG - Open Angle Glaucoma}

The ordinary form of Glaucoma is open-angle Glaucoma (Fatima et al., 2017). It contrasts the angleclosure red Glaucoma in which the drainage angle among the cornea and the iris becoming closer; it blocks fluid from the eye and results in a significant rise in the pressure.

In Glaucoma (Fig. 2), the decay of fiber nerves causes cup size to increase, and as resultant, the CDR also increases. CDR is one of the main clinical indicators involved in glaucoma diagnosis. Accuracy of cup diameter is directly proportional to the Internal Limiting Membrane (ILM) layer, as CDR is our main feature. That's why we have extracted the ILM layer. Accuracy of disc diameter is directly proportional to the RPE layer, as CDR is our main feature; this motivates us to extract the RPE layer. We have selected the OCT images as subject while major prior published studies have experimented on fundus images.

Figure 2. Real Image of Glaucomatic Eye

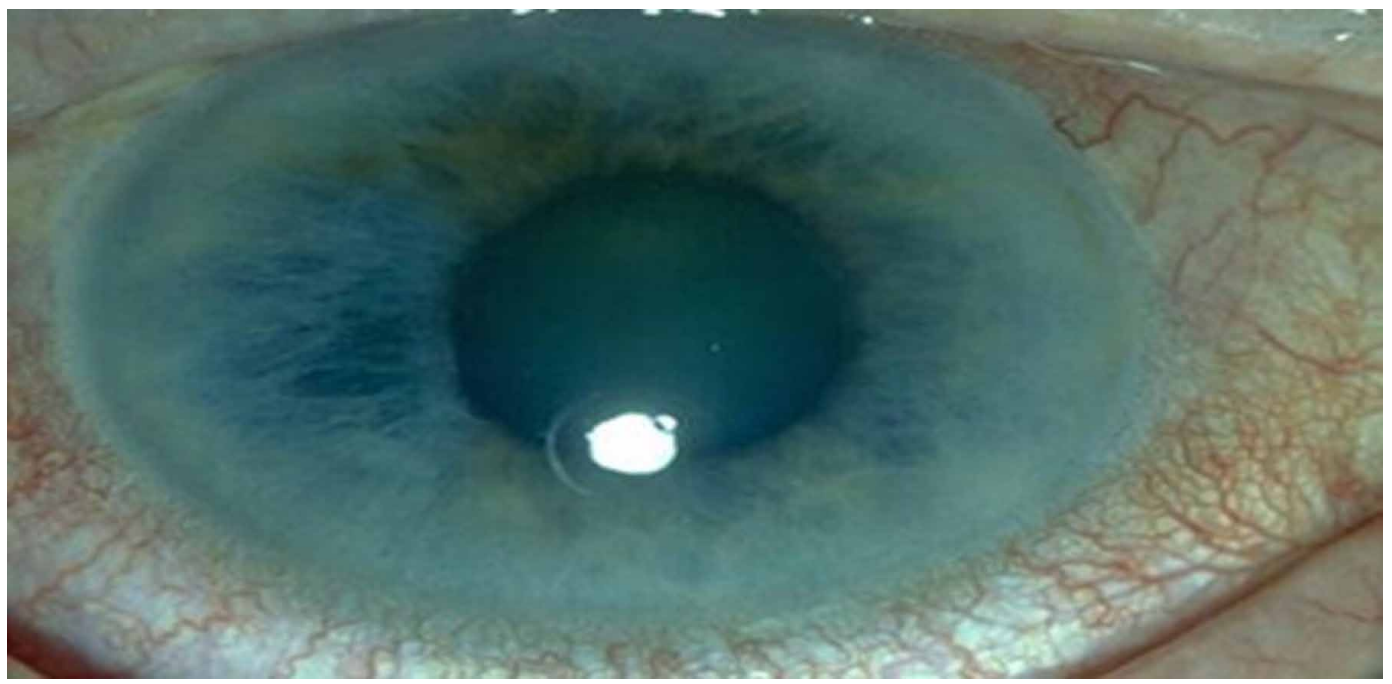

\subsection{Motivation and The Proposed Work}

Glaucoma is also called silent theft of sight, which results in the irreversible vision loss due to damage in the optical nerve of an eye. The only available alternative is the early and timely diagnosis of the disease so that the proper treatment can be started so as to slow down the progression. Ophthalmologist diagnoses this disease using a retinal examination of the dilated pupil. They use different comprehensive retinal examinations such as ophthalmoscopy, tonometry, perimetry, gonioscopy and pachymetry to diagnose glaucoma. But all these approaches are manual, time-consuming and may be prone to subjective errors. Highly experienced and expert Ophthalmologists are required for precise diagnosis which leads to the overburden. This all motivates the researcher's fraternity to develop a fast, accurate and automated approach in the field of medical imaging for analysis of the retinal images for glaucoma diagnosis. With the intrusion of techniques like machine learning in the domain of medical imaging, 
enormous modifications have been observed in the classical techniques of diagnosis of diseases like glaucoma. The proposed empirical study is a generic attempt to develop robust and highly predictive machine learning based computer-aided diagnosis system that may aid as an assistive measure for the initial screening of glaucoma for diagnosis purposes by classifying the retinal images as "healthy" or "glaucomatous". 40 ORB features and 5 well accepted vital features are selected for this work to classify the retinal OCT images of potential patients. The proposed system could serve as a second opinion for ophthalmologists and at the same instant it will also reduce burden on already overloaded experienced ophthalmologists and will help humanity.

The machine learning system had an excellent ability to work on medical imaging classifications. This inspires us to build a proposed system which will support to enhance the standards for glaucoma diagnosis and improve diagnosis consistency. We have extracted five features Retinal Pigment Epithelium (RPE) layer, ILM Layer, Cup Diameter, Disc Diameter, and CDR ratio by self crafted algorithms and the remaining forty features were extracted using the ORB algorithm. In this empirical study, additional experiments were performed to validate the system for comparison, we also extracted OCT parameters and among these parameters average thickness, CDR ratio was calculated.

These self crafted five algorithms for extracting features from the OCT image are easy to understand and implement. The quality of the image does not cause any effect on our algorithm; they still compute an accurate result.

Going through various search engines we have identified the research gap in this domain, as per the best of the author's knowledge, very few reputed studies are recently (or may be in past also) published on glaucoma identification using OCT images, with the support of machine learning based classifiers. Hence we are the front runners to propose such a type of, first, study with OCT images, these forty-five parameters, and the selected five benchmarked machine learning algorithms.

The organization of this paper is started with the introduction of the type of disease in retinal (eyes) along with the differentiation of normal eye and Glaucoma infected eye. A detailed description of OCT and various shortlisted machine learning are also presented. Further, Section 2 discusses the background and related work in the field of Glaucoma OCT images. In Section 3, a brief outline is provided for the proposed methodology and various feature extraction techniques required for the diagnosis of Glaucoma in retinal OCT. Section 4 reports the performance measurement of different machine learning algorithms for the mentioned purpose along with result discussion followed by the conclusion at the last Section 5.

The significant contributions of this paper include the following purpose and objective:

- To propose the procedures for extracting various features like - RPE, ILM layer, Cup Diameter, Disc Diameter, and CDR ratio from the OCT image and thereafter performing experiments using these features.

- Filling up the research gap by proposing the first study, of its own kind, where the objective is categorization of OCT images into two separate classes; in which machine learning algorithms accept input as values of 45 vital features extracted from OCT images.

- To provide a comparative study of the simulated results when experiments were performed on customized dataset.

- To perform the prediction of glaucoma with classification accuracy of SVM, KNN, Random Forest, LDA, and Decision tree as $75 \%, 97 \%, 88 \%, 92 \%$, and $92 \%$, respectively.

- Encouraging findings are observed that the KNN model gives the best performance based on the accuracy, sensitivity and specificity of the classifier, as $97 \%, 100 \%$ and $85.71 \%$, respectively. 


\section{LITERATURE SURVEY}

For this section, we have shortlisted some of the prominent prior published studies, including latest ones, where researchers have attempted to solve the problem in hand. Finally, a comparative table is also presented for readers.

Khalil et al.,2018 experimented on SD-OCT images; they have used ILM (inner limited membrane) as a parameter which gives accurate results even in the poor visualization. They use the ILM process to generate solid contour that is used to generate accurate cup diameter. In this study, a technique has been proposed to examine the retinal layer thoroughly for accurate results, it provides the latest approach to contract with the sharp cases. Practitioners (Pavithra et al.,2019) in their study have used SD-OCT for the diagnosis of prime open-angle Glaucoma \& normal Glaucoma by considering macular thickness, ganglion cell complex and RNFL thickness. The learning of the neural network is done in such a mode that the ANN would be able to detect so many glaucoma parameters in all different levels using the training process. The proposed model was built-up with the help of a feedforward backpropagation network in the neural network domain. Authors (Fathima cs et al.,2010) suggest that Fundoscopy and OCT are the best options for glaucoma detection. Through this, they calculate the Cup to disc ratio. Researchers have applied K-mean clustering and Otsu thresholding.

Practitioners (Amina Jameel and Imran Basit,2018) in their empirical study, give a fully automatic and simultaneous method by utilizing domain knowledge and inexpensive computational methods to segment 5 layers from an OCT image. Fuzzy histogram hyperbolism is used to transform the distinct ROI to improve the homogeneity within individual layers. They select the unique hyper-reflective layers of the retina, which are integrated into the continuous max-flow graph cut framework for robust segmentation to compute the desirable data term. Anushika Singh et al., 2019 attempted for detection of Glaucoma, where automated image analysis was developed using wavelet features from the segmented optic disc. The proposed algorithm was the way of glaucoma recognition that contains an analysis of wavelet features in the segmented optic disc image.

In this study (Lee et al.,2018) the Otsu's thresholding is used as the conversion of greyscale images into the binary images. This method mainly takes the images in only the two groups of pixels and it follows the bi-modal histogram method (foreground and background pixel). The calculation of threshold for optimization in which they separate these into two groups so that their mutual result extends is equal or can be minimized and also their inter-class variance is high.

In the experimental study, by Won et al.,2016, the Gray Scale Image (GSI) of all the green channels are using the CDC process of ILM-layers extractions. The ILM-layer iteration processing needs to apply for boosting the ILM layer on Gray Scale Image. After that, the thresholding process is applied to segments retinal layers through all scale images. All the top profiles in the segmented retinal layer had computed to extract the ILM layer. Next, the ILM layer peak Surface (TSILM) should be refined by using the set of future novel techniques. At last, the cup edge has placed in the developed top profile based on the endpoint of the RPE-layer. The study by (Bizios et al.,2010) uses both ganglion cell inner plexiform layer and retinal nerve fiber layer patterns of glaucoma progressions by determination using SD-OCT guided progression analysis software. In this empirical study, by Wollstein et al.,2005 OCT is used as the powerful tool which is used for Glaucoma with both qualitatively \& quantitatively and targeted the optic disc \& macular area, it can be reveal preperimetric glaucomic eyes with high sensitivity \& specificity. It calculates the retinal nerve fibre layer thickness and ganglion complex cell thickness.

This study, Bussel et al., 2014 uses the technology to detect optic disc boundary and for this purpose image preprocessing is introduced. Initially, the localization of the region of optic disc is to be presented by using the red channels. These red components have been utilized like it is established to have the big difference among all the non optic disc \& optic disc areas in comparison with the other channels. For the removal of the blood vessels, the morphological closing operations have been done. In this study, An et al.,2019 Retinal Nerve Fiber Layer, Optic Nerve Head and Macular thickness are 
measured for detecting Glaucoma in OCT. ROC curve and sensitivities were calculated . Combination of RNFL and ONH (Optic nerve hypoplasia) proved to be the best parameter.In the research paper, by Koh et al., 2018, OCT was used for the glaucoma diagnosis and screening of glucomatic progression. The commonly available iterations of OCT technology, spectral domain (OCT), have advantage in the glaucoma assessments. In this authors have presented a number of commercially existing SD-OCT devices with different parameters and the distinctive features.

In this next experimental study researchers, Anusorn et al.,2013 observed that the fuzzy logic and image processing algorithms is a novel expert scheme for early detection of Glaucoma. An experimental result shows the proposed method superiority. It is mainly based on segmentations of $\mathrm{OD}$ (optic disc) and OC(optic Cup) which is also presented for the identification of Glaucoma and the segmentation is perform by the use active contour mode.

Chakraborty, 2019 proposed an automated computer-aided chronic wound diagnostic system for wound tissue characterization precisely which can address a large volume of patients. Fuzzy c-means clustering along with four different computational learning schemes viz., Naïve Bayes, Linear discriminant analysis, Decision tree, and Random forest, have been presented to achieve automated wound tissue identification for pressure and diabetic ulcer diagnosis. The random forest approach provided the highest classification accuracy when classifying wound patterns with color features extracted from the segmented portions of image data sets. In the another study by the same author, Chakraborty, 2019, analyzed a set partitioning in hierarchical tree (SPIHT) compression technique for chronic wound image transmission under smart phone-enabled Tele-wound network system. The proposed system aids in heavy volume of best quality wound images to be compressed, processed and sent over wireless media with very low bandwidth and reliable networks.

In very recently published two studies on glaucoma; in first study authors (shehryar et al., 2020) focused on detection of glaucoma by combining and correlating fundus and SD-OCT image analysis. In the second study the authors(Ajest et al., 2020)attempted to increase the accuracy of glaucoma detection by proposing a novel an effective optimization-driven classifier. The proposed Jaya-CSO is designed by integrating the Jaya algorithm with the chicken swarm optimization (CSO) technique for tuning the weights of the RNN classifier.

In this final selected study practitioners, Hassan et al.,2015 observed that Retinal nerve fiber layer and fast retinal nerve fiber layer is used as a parameter and for the proposed methodology visual field testing used considering RNFL to differentiate between healthy and glaucomatous eye, and the result shows that both the parameters gives same accuracy.

Given below Table 1 presents the detailed description of 13 shortlisted prior published literatures (some of the studies are recently published) on the basis of various parameters like dataset size, technique used, scan region selected, accuracy etc.

\section{PROPOSED METHODOLOGY}

\subsection{Objective of This Study}

Glaucoma is the most common disease that leads to vision loss (CS,2019). This disease harms the optic nerve of the eye, and it causes ultimate blindness. It raises intraocular pressure which results in the increment of Cup to disc ratio if it is not treated at an early stage. The imperative intention of this study is to search for a methodology to explore and identify Glaucoma during the early stage. In the scarcity of glaucomatous experts, and automatically Glaucoma detected system, the proposed system is advantageous.

As the initial step of the preprocessing stage, we initiate with the segmentation of the region of interest (ROI) by putting the value of the cup precisely in the middle with some weights of layers of the retina on both sides.In the preprocessing of the OCT image, we have attempted to improve the 
Table 1. Table portraying comparison among prior published studies on various parameters

\begin{tabular}{|c|c|c|c|c|c|}
\hline Study and reference & Data sets & $\begin{array}{c}\text { Technique } \\
\text { Used }\end{array}$ & $\begin{array}{l}\text { Features } \\
\text { Extracted }\end{array}$ & Classification & Accuracy \\
\hline (Khalil et al.,2018) & $\begin{array}{l}\text { Glaucoma } 63 \\
\text { Healthy } 150\end{array}$ & $\begin{array}{c}\text { Optical } \\
\text { Coherence tomography }\end{array}$ & $\begin{array}{l}\text { ONH, } \\
\text { RPE,RFN layer, } \\
\text { CDR } \\
\text { comparision }\end{array}$ & NIL & $94 \%$ \\
\hline (Pavithra et al., 2019) & $\begin{array}{c}\text { Normal } 52 \\
\text { PAOG } 61 \\
\text { NTG.MT } 56\end{array}$ & Back Propagation & $\begin{array}{l}\text { RNFL } \\
\text { Thickness }\end{array}$ & $\begin{array}{l}\text { ANN } \\
\text { KNN }\end{array}$ & $91 \%$ \\
\hline $\begin{array}{c}\text { (Fathima cs et al } \\
., 2017)\end{array}$ & $\begin{array}{c}\text { Glaucoma } 110 \\
\text { Healthy } 205\end{array}$ & $\begin{array}{c}\text { K-means clustering } \\
\text { and Otsu thresholding } \\
\text { techniques }\end{array}$ & $\begin{array}{l}\text { SD-OCT, } \\
\text { GCC, } \\
\text { CDR, } \\
\text { IOP, } \\
\text { CDR, } \\
\text { RDR }\end{array}$ & ANN & $84 \%$ \\
\hline $\begin{array}{c}\text { (Amina Jameel et } \\
\text { al.,2018) }\end{array}$ & $\begin{array}{c}\text { Glaucoma } \\
\text { Opt1 } 27 \\
\text { Opt2 } 26 \\
\text { Opt3 } 23 \\
\text { Opt4 } 24 \\
\text { Healthy } \\
\text { Opt1 } 23 \\
\text { Opt2 } 24 \\
\text { Opt3 } 27 \\
\text { Opt4 } 26\end{array}$ & $\begin{array}{l}\text { Interpolation } \\
\text { Bezier curve }\end{array}$ & $\begin{array}{l}\text { SD-OCT, } \\
\text { ONH, } \\
\text { ILM-LAYER }\end{array}$ & $\begin{array}{c}\text { Nerve Fiber } \\
\text { Layer } \\
\text { Ganglion Cell } \\
\text { Outer Plexiform } \\
\text { Layer } \\
\text { Outer Nuclear Layer }\end{array}$ & $\begin{array}{l}\text { ADHF-80\% } \\
\text { ADSN- } \\
96 \%\end{array}$ \\
\hline $\begin{array}{c}\text { (Anushika singh et } \\
\text { al.,2019) }\end{array}$ & $\begin{array}{l}\text { Glaucoma } 63 \\
\text { Healthy } 79\end{array}$ & $\begin{array}{l}\text { Prominent Feature } \\
\text { Reduction using Principal } \\
\text { Component Analysis) }\end{array}$ & CDR & $\begin{array}{l}\text { KNN } \\
\text { ANN } \\
\text { SVM }\end{array}$ & $87 \%$ \\
\hline (Lee et al.,2018) & $\begin{array}{c}\text { Glaucoma } \\
\text { Mild } 162 \\
\text { Moderate } 42 \\
\text { Healthy } 112\end{array}$ & $\begin{array}{l}\text { Random Forests method } \\
\text { RTVue } \\
\text { SD-OCT }\end{array}$ & $\begin{array}{c}\text { Macular RNFL } \\
\text { ONH } \\
\text { cpRNFL } \\
\text { mRNFL } \\
\text { GCIPL } \\
\text { Macular } \\
\text { ganglion cell }\end{array}$ & NIL & $92 \%$ \\
\hline (Won et al., 2016) & $\begin{array}{l}\text { Open-angle } \\
\text { Glaucoma } 79\end{array}$ & $\begin{array}{l}\text { SD-OCT's guided } \\
\text { progression analysis } \\
\text { software }\end{array}$ & $\begin{array}{l}\text { RNFL, } \\
\text { SD-OCT, } \\
\text { GCIPL, } \\
\text { Macular } \\
\text { RNFL }\end{array}$ & NIL & $\begin{array}{l}\text { Upto } 90 \% \\
\text { (Opt4) }\end{array}$ \\
\hline (Koh et al., 2018) & $\begin{array}{c}\text { Glaucoma } 49 \\
\text { Healthy } 43\end{array}$ & $\begin{array}{c}\text { Spectral Domain- } \\
\text { scanning Laser } \\
\text { Ophthalmoscopy,Ocular } \\
\text { SD-OCT }\end{array}$ & $\begin{array}{c}\text { Bland altman } \\
\text { plots average } \\
\text { Thickness, } \\
\text { Retinal nerve } \\
\text { Fiber Layer } \\
\text { Thickness }\end{array}$ & NIL & $\begin{array}{l}\text { ANN }-95 \% \\
\text { SVM- } 94.5 \%\end{array}$ \\
\hline (Anusorn et al., 2013) & $\begin{array}{c}\text { Glaucoma } 58 \\
\text { Healthy } 67\end{array}$ & $\begin{array}{l}\text { Image Processing } \\
\text { Technique of Automated } \\
\text { Glaucoma detection }\end{array}$ & $\begin{array}{l}\text { Tonometry } \\
\text { Opthalmoscopy } \\
\text { Perimetry } \\
\text { Ganioscopy, } \\
\text { CDR }\end{array}$ & $\mathrm{CDR}$ & $80-95 \%$ \\
\hline (Hassan et al., 2015) & $\begin{array}{c}\text { Glaucoma } 73 \\
\text { Healthy } 77\end{array}$ & SD-OCT & $\begin{array}{l}\text { ONH, } \\
\text { RNFL,ONH } \\
\text { GCC }\end{array}$ & NIL & $75 \%$ \\
\hline $\begin{array}{c}\text { (Burgansky et } \\
\text { al.,2005) }\end{array}$ & $\begin{array}{c}\text { Glaucoma } 76 \\
\text { Healthy } 90\end{array}$ & $\begin{array}{l}\text { Fuzzy logic and image } \\
\text { processing }\end{array}$ & $\begin{array}{l}\text { ONH, } \\
\text { CDR }\end{array}$ & NIL & $78 \%$ \\
\hline (Bock et al., 2010) & $\begin{array}{c}\text { Glaucoma } 50 \\
\text { Healthy } 80\end{array}$ & Sirus SD-OCT & $\begin{array}{l}\text { Macular ONH, } \\
\text { Macular } \\
\text { RNFL, disc } \\
\text { topography, } \\
\text { circumpapillary } \\
\text { RNFL } \\
\text { thickness, } \\
\text { macular } \\
\text { RNFLT, } \\
\text { cpRNFLT. }\end{array}$ & NIL & $84 \%$ \\
\hline (Xu et al., 2011) & $\begin{array}{c}\text { Glaucoma } 65 \\
\text { Healthy } 89\end{array}$ & $\begin{array}{l}\text { mean deviation and visual } \\
\text { field index }\end{array}$ & $\begin{array}{c}\text { RNFL } \\
\text { thickness, } \\
\text { Retinal nerve } \\
\text { fiber layer } \\
\text { macular } \\
\text { ganglion cell }\end{array}$ & NIL & $90 \%$ \\
\hline
\end{tabular}


quality, which is required to design an algorithm to measure the thickness of ILM, RPE layer, and Cup to disc ratio of an OCT (Fig. 3) image from the Mendeley dataset and own created one.

\subsection{Methodology}

The classification accuracy of the algorithms is more sensitive to the training datasets(SalazarGonzalez et al., 2014). Hence, the researchers must consider the real-time scenario while selecting the datasets for the training. That is why our dataset consists of a combination of private and public images of OCT images. Mendeley dataset(Raja et al., 2020) was collected for making research easier on diagnosis of Glaucoma using computer-aided systems. The resolution of images is $200 * 200$ pixels (Fig. 2). The images were contributed by four ophthalmologists distinctively. All ophthalmologists have annotated the CDR ratio very precisely. The dataset contains both healthy and glaucomatous OCT (Fig. 3) images. Fig. 4 demonstrates the ILM layer, RPE, Cup Diameter, and Disc Diameter in OCT images and Fig. 5 demonstrates the thorough description of the proposed process implemented in this experimental study. The use of the smaller training data at the training phase reduces the classification accuracy. Hence, more features need to be used(Zilly et al., 2017), that is why we have collected a sufficient number of 45 vital features through two approaches. These features are supplied to five well-known machine learning classifiers (SVM, KNN, LDA, XGBoost and Random forest)

Figure 3. OCT Image

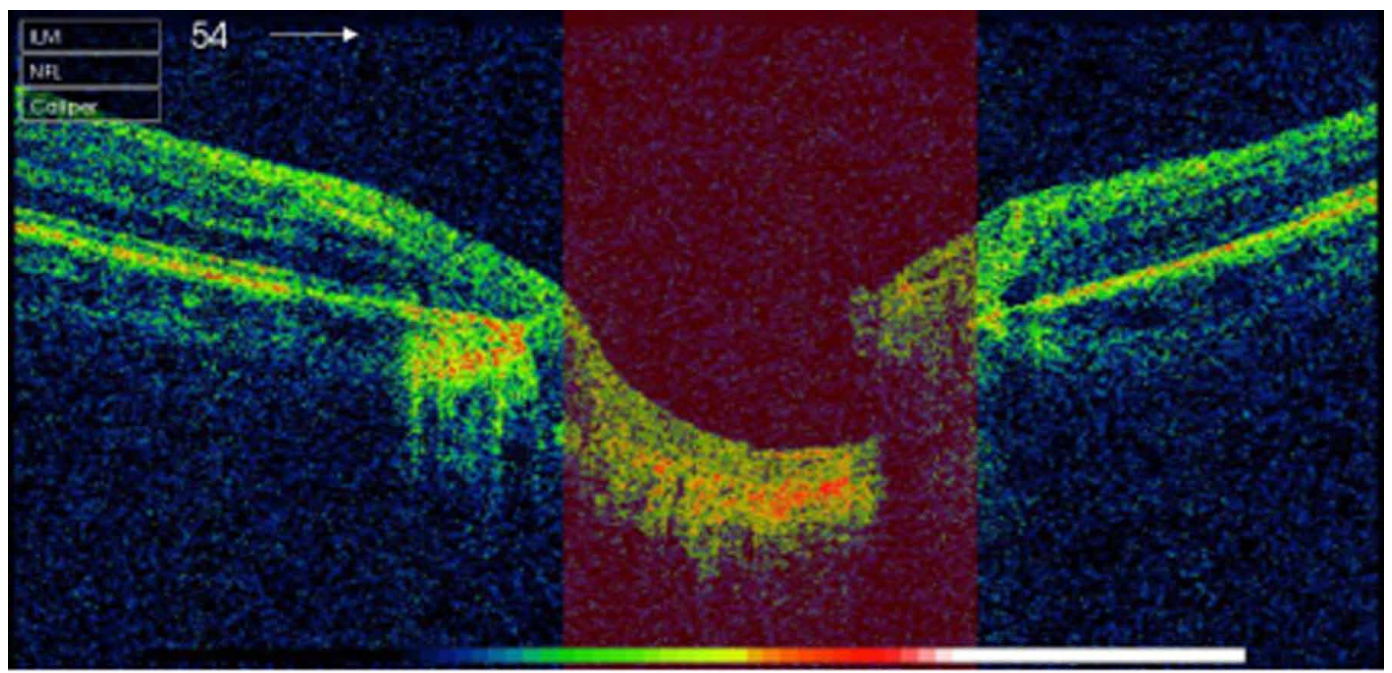

for classification.

\subsection{Preprocessing}

Preprocessing is implemented to enhance the quality of the images. It includes resizing and rescaling an image. Images of dimensions $200 * 200$ were selected, which were rescaled by four times the original image. This study comprises of morphological operation as data preprocessing techniques (Fig. 5).

\subsubsection{Morphological Operation}

Morphology is an operation that processed images based on shape (Fig. 6).

The morphological operation has the following positives: 
Figure 4. ILM, RPE, Cup Diameter and Disc Diameter in OCT images
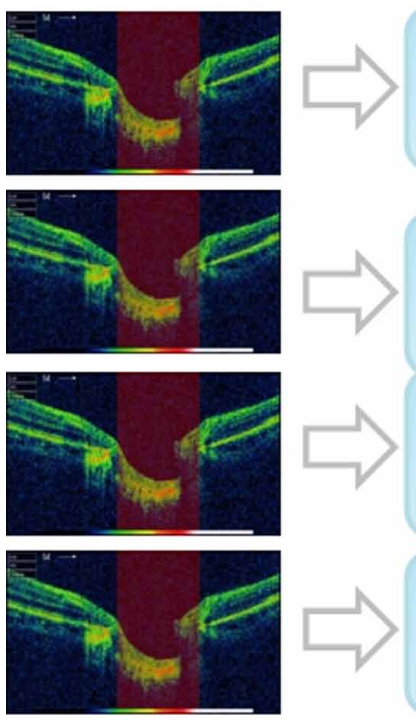

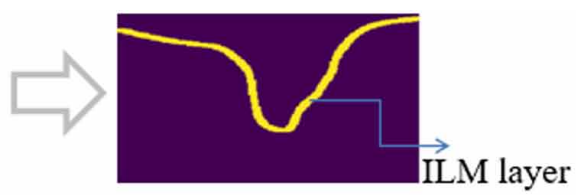

RPE Extraction

Algorithm

Cup diameter

Extraction

Algorithm

Disc diameter

Extraction

Algorithm
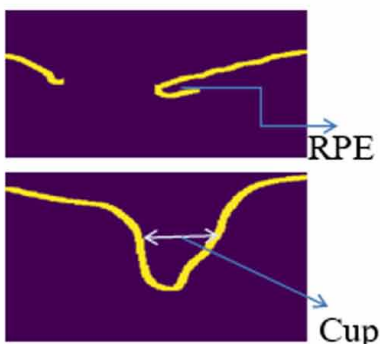

Cup Diameter

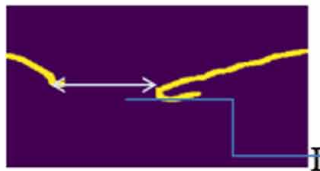

Disc Diameter

Figure 5. Complete diagram of the proposed system for glaucoma detection

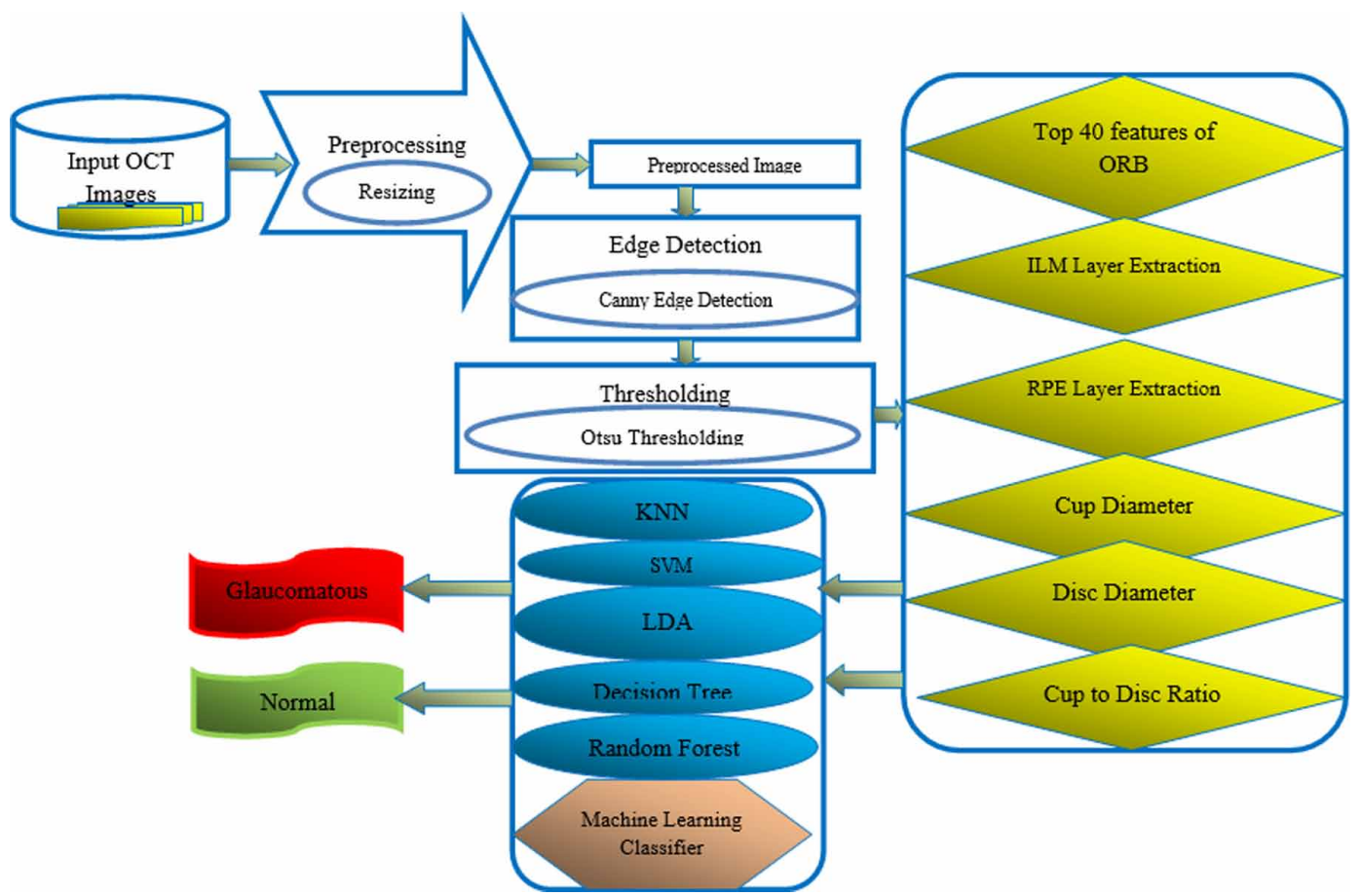

- It processes an image pixel by pixel.

- It effectively highlights the required features and removes unwanted features

- Minor elements can be removed without increasing the size of the structure. 


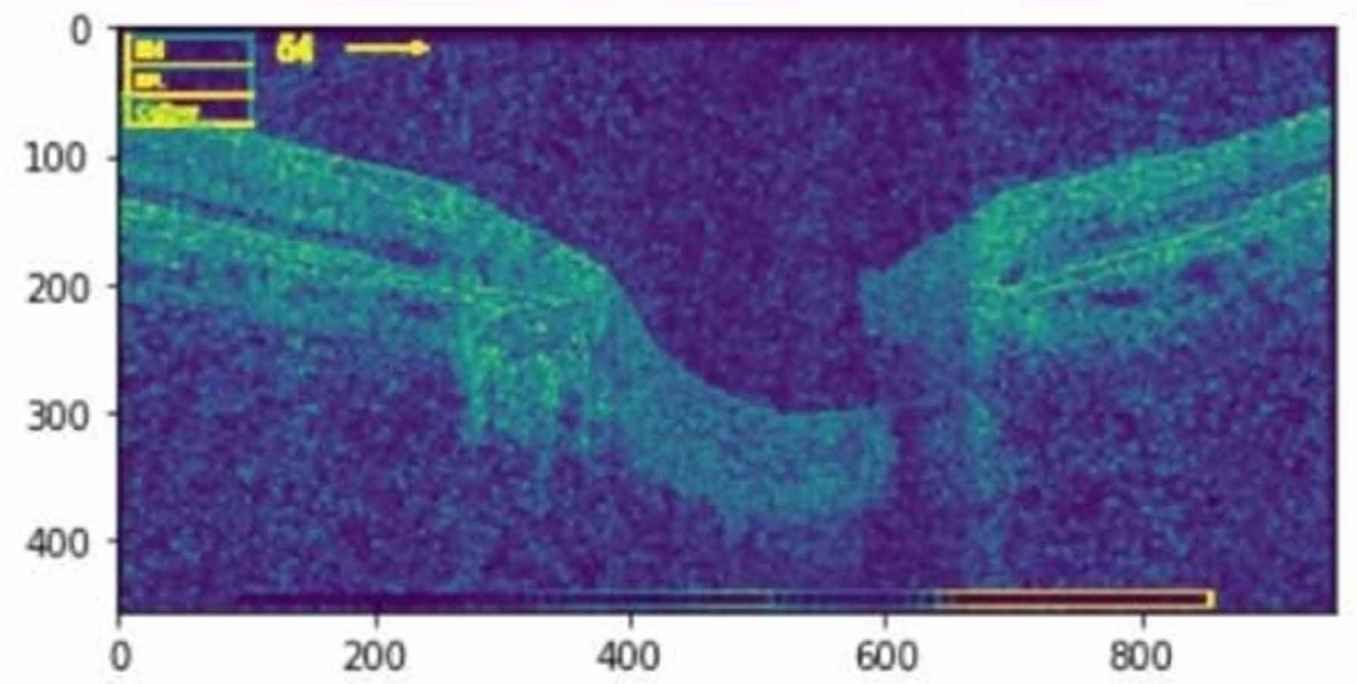

In this study, we have used an element of structuring for an input image, and then it produces an output image. The most basic leveled operations are erosion and dilation. Erosion gradually removes away the boundaries of foreground objects. It is used to diminish the features of an image. Dilation increases the object area and is used to accentuate features.

\subsection{Edge Detection}

An edge detector gives us a binary image as an output. Edge detector can perform the following functions like Noise Detection, Gradient Calculation and Edge Tracking. The edge detector argument is called sigma. Decreasing the value of sigma gives more number of edges. This study allows sigma equals to 3 .

For implementation, a Canny edge detector is selected, for this study, which is the technique that is used to extract structural information and remove the data amount to be processed. It explores a wide range of edges in images. It includes Gaussian filters, which reduce the effect of noise available in the image (Fig. 7). It has three parameters (the noise, the images, and the higher width).

Figure 7. Edge Detection
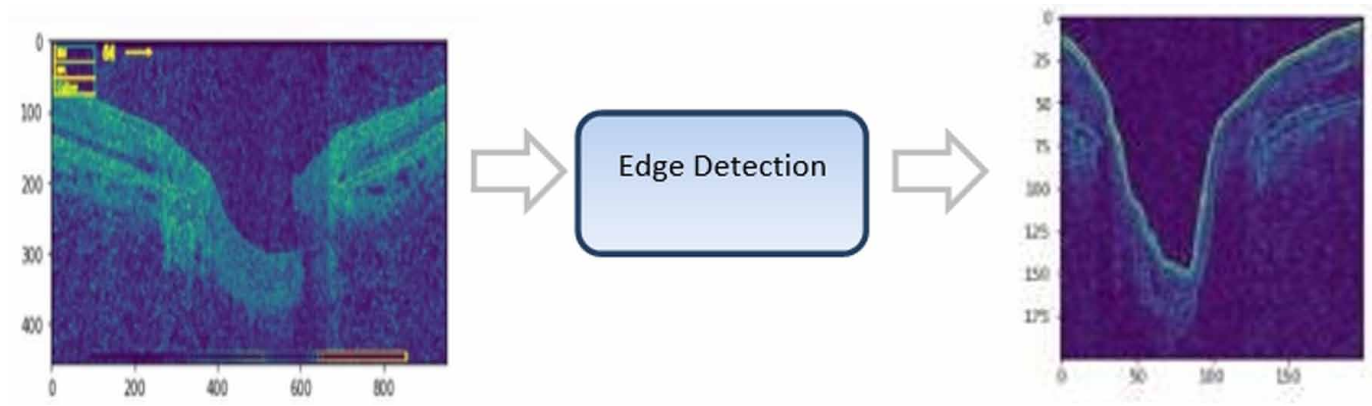


\subsection{Thresholding}

Thresholding divides the pixel into two classes foreground and background. It is used for converting the images, likewise, grayscale into a binary image. The set of values of the threshold is wholly based on the images of the level of gray distribution by using multiple levels of the Otsu algorithm.

By thresholding, we get the desired area. By applying various thresholding, we get our desired output to Otsu thresholding. For further implementation, this study uses Otsu thresholding (Fig. 8). The value of Otsu is used to minimize the intraclass variance of the input image, which has been selected.

Figure 8. Morphological Operation, Thresholding, Edge Detection
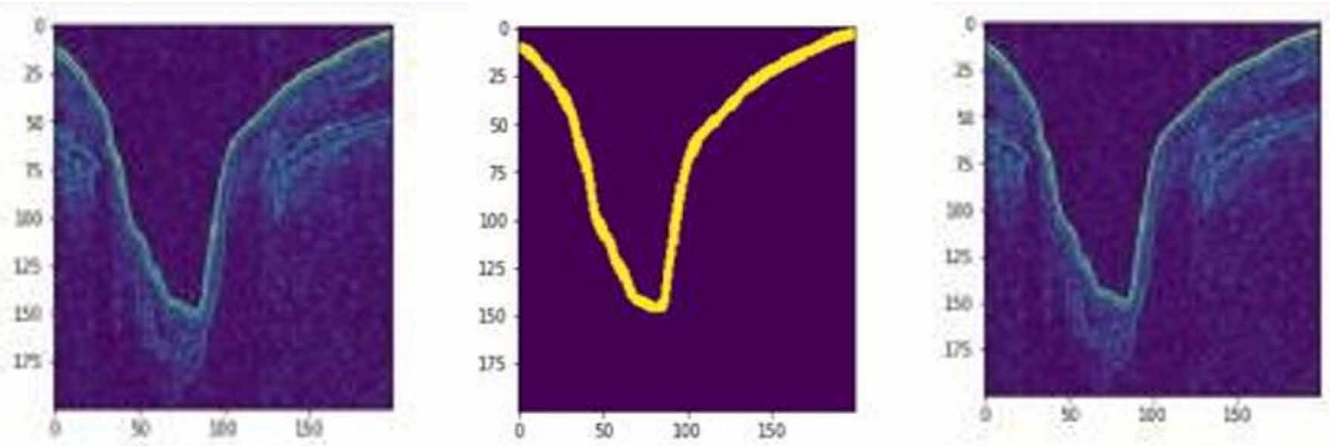

\subsection{Feature Extraction}

\subsubsection{ORB}

ORB(Deepanshu Tyagi,2019) is the addition that is used for fast and accurate orientation components. It causes better performances in the closest applications of neighbors. Functions of ORB algorithm use a multi-scale image pyramid because fast features don't have the component of orientation and multi-scale features. The image of different resolutions is a multi-scale representation image pyramid. Binary Robust Independent Elementary Feature (BRIEF) represents an object by taking all the key points molds by the fast algorithm and transforms it into a binary feature vector. In detail, each key point is explained by a feature vector, which is $128-512$ bits string. ORB algorithm sum up the main direction by excellent centroid method represents the centroid in the local tile region of the feature point and then builds direction vector that points from image block.

$$
m_{p q}=\sum_{a, b} a^{p} b^{q}
$$

$\mathrm{p}, \mathrm{q}$ represents the dimension of the image, a,b represents the coordinate of image, $\mathrm{k}(\mathrm{a}, \mathrm{b})$ represents the intensity of the image and $m_{p q}$ represents the centroid of the image.

$\mathrm{K}(\mathrm{a}, \mathrm{b})=$ gray value of point $\mathrm{a}, \mathrm{b}$.

$m_{p q}=\sum_{a, b} a^{p} b^{q}$

The image of centroid block can be acquired by moment 
$x=\left(\frac{m_{10}}{m_{00}}, \frac{m_{01}}{m_{00}}\right)$

$m_{00}=$ Zero moment, $m_{10}=$ First Moment

$m_{01}=$ First Moment, $x=$ Centroid of an image

$\alpha=a \tan 2\left(m_{01}, m_{10}\right)$

$\alpha=$ Angle between moments

In this process, the moment is calculated to get the changes occurring in an image. It is difficult to identify changes that are occurring in an image by just checking its pixel value. Therefore, the calculation of the moment is done.

In equation (1): $\mathrm{k}(\mathrm{a}, \mathrm{b})=$ Intensity of any pixel, $\mathrm{a}$ and $\mathrm{b}$ are coordinate position(Rublee et al., 2011).After calculating the moment, the centroid value gets calculated so that there is no need to work with an individual pixel.

In equation (2): $\mathrm{m}_{00}$ (zero moments) is the summation of all intensities of a pixel in an image.

$\frac{m_{10}}{m_{00}}$ (First moment) summation of the product of the intensity of all pixels and its corresponding points and $\mathrm{x}=$ centroid.

The angle between the moment is calculated in equation: $\alpha=$ angle.

Oriented FAST is having a fixed value, which takes a circle with radius 3 . The distant point seems to be the corner of the ORB due to the scale point, and may not be cleared.ORB algorithm is executing by building an image pyramid, and finding corner points on each layer of the pyramid is open $\mathrm{cv}$.

\subsubsection{Internal Limiting Membrane(ILM) Layer Extraction}

The ILM (Fig. 9) is the basal lamina of the inner retina and boundary between the retina and vitreous body by astrocytes and Muller cells. It is a lean and translucent cellular covering on retina's plane. The outer surface of this membrane is uneven, and the inner surface is smooth. The ILM(Gelman et al., 2015) of the retina is continuous with the internal membrane of the ciliary body. It is formed by the expansion of the fibers of the optic nerve. ILM layer Extraction is an essential part of the CupDiameter-Calculation process. ILM is the last layer before the background. The purity of the cup diameter is directly proportional to ILM layer extraction; slight changes cause incorrect results. In our scrutiny, we proposed a unique method to facilitate accurate results (Figure 9).

Algorithm 1 ILM Extraction (Refer Figure 10)

Step 1: START

Step 2: Initialize white as True

Step 3:Initializeblack as False

Step 4: For each pixel in ILM(for $i=0$ to $w$, where $w$ is the width

of the image)

Step 4.1: For each pixel in ILM (for $j=0$ to $h$, where $h$ is the

height of the image)

Step 4.2:IF ILM[j, i]==black then

i. Continue

Step 4.3: END IF 


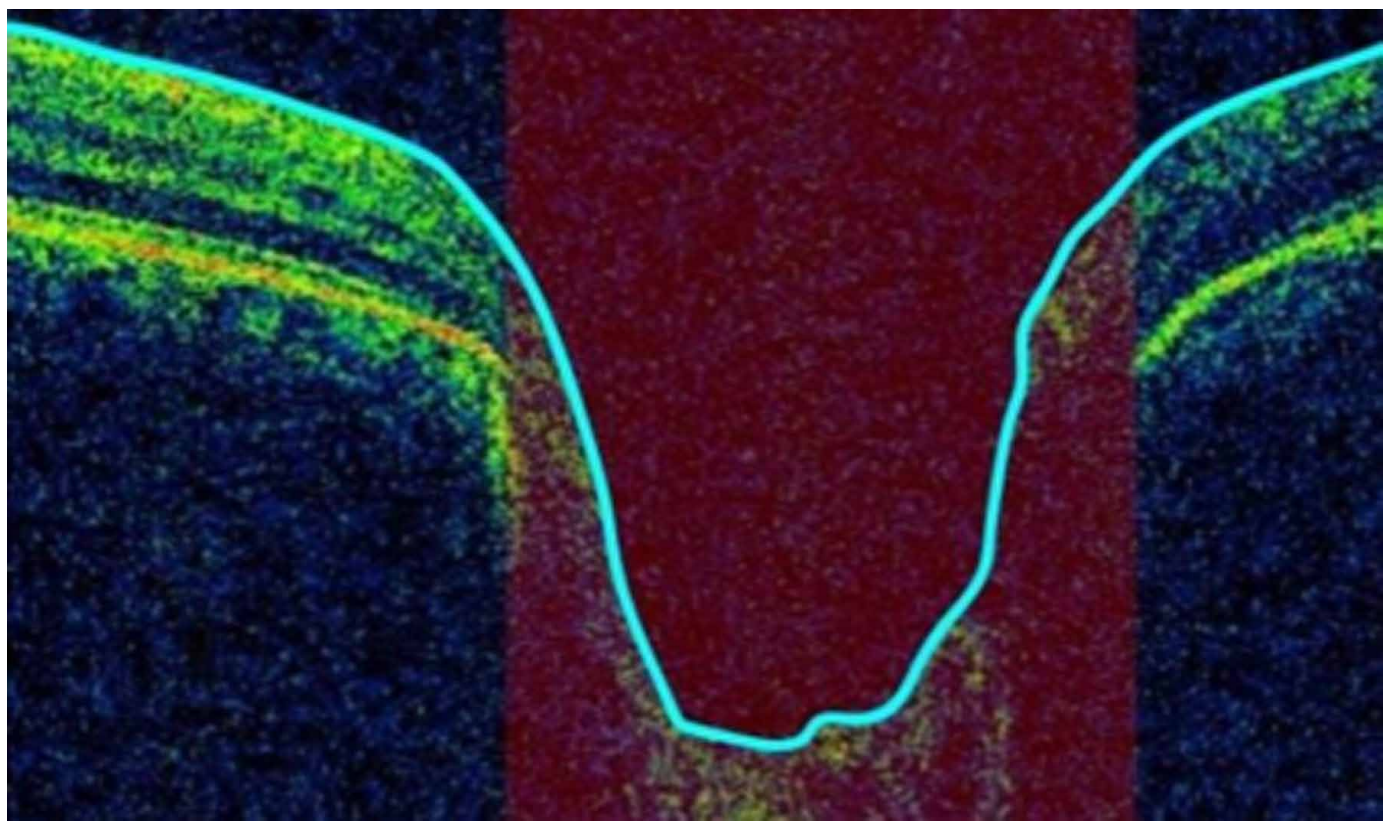

Step 4.4: IF ILM $[j, i]==w h i t e$ then

ii. WHILE $(j !=w$ and $\operatorname{ILM}[j, i]==w h i t e)$

iii. increment $j$ by 1

iv. For each pixel in ILM (for $k=j$ to $h$ )

$\mathrm{v}$. set ILM[k,i] to black

vi. END WHILE

Step 4.5: END IF

Step 5: STOP

\subsubsection{Retinal Pigment Epithelium(RPE) Layer Extraction:}

RPE layer (Fig. 11) is the second last retinal layer. This layer has a neurosensory retina that nourishes visual cells and is only attached to underline choroid and underline retinal visual cells.

Figure 10. ILM Layer
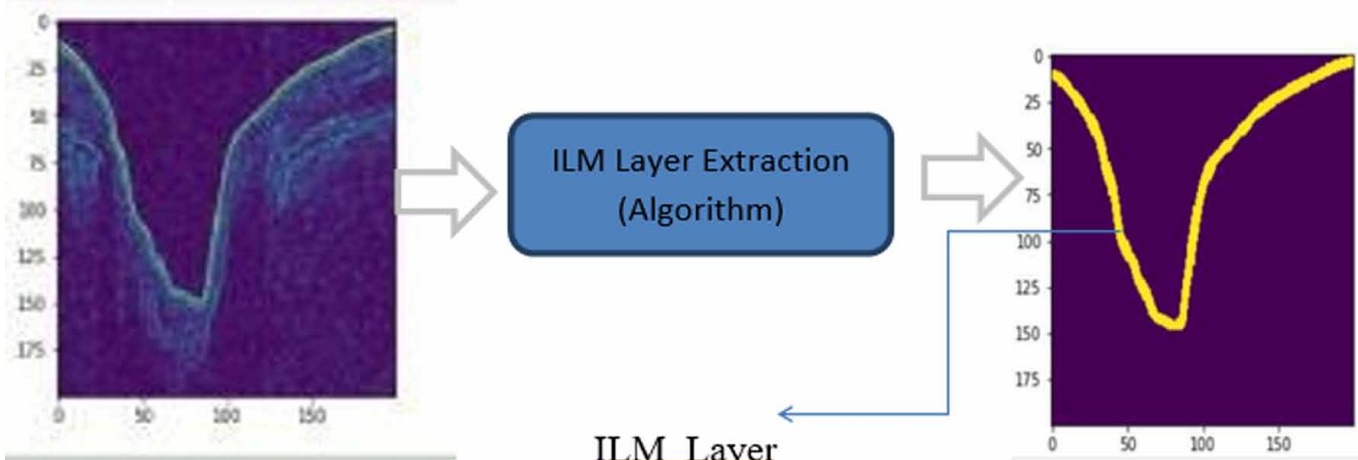

ILM Layer 


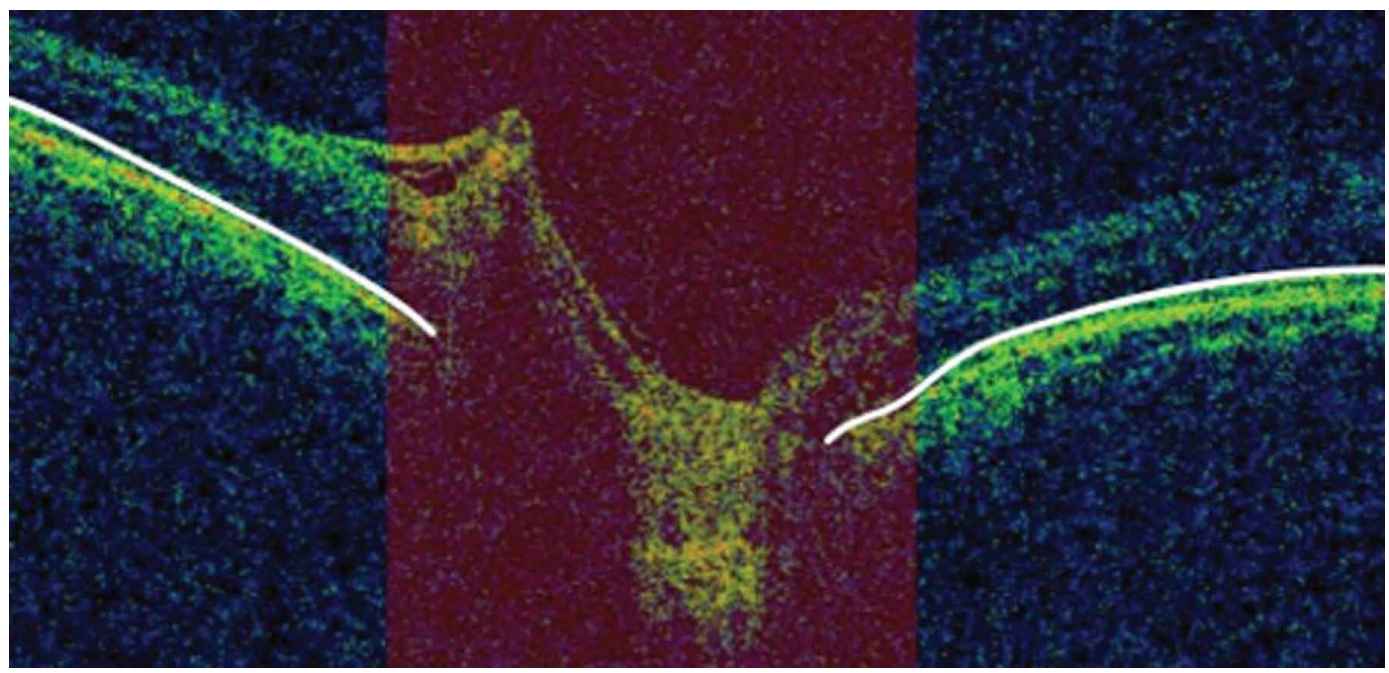

The RPE(Boulton and Dayhaw-Barker,2001) is the continuous monolayer of cuboidal post-mitotic epithelial cells, which is located in between the photoreceptors and Bruch's membrane. Some functions of the RPE layer are light absorption, visual cycle, secretion, and immune modulation, etc.

These functions of RPE depend on the address of the retina and changes related to ages. Pigmentation of RPE changes with age. It is having a single layer of hexagonal cells. RPE gives the norm environment for the creation of reactive oxygen species with the power for damaging proteins, DNA, and lipids. To find the RPE layer, bottom layers classified into two folds, and the top fold is called an RPE layer.

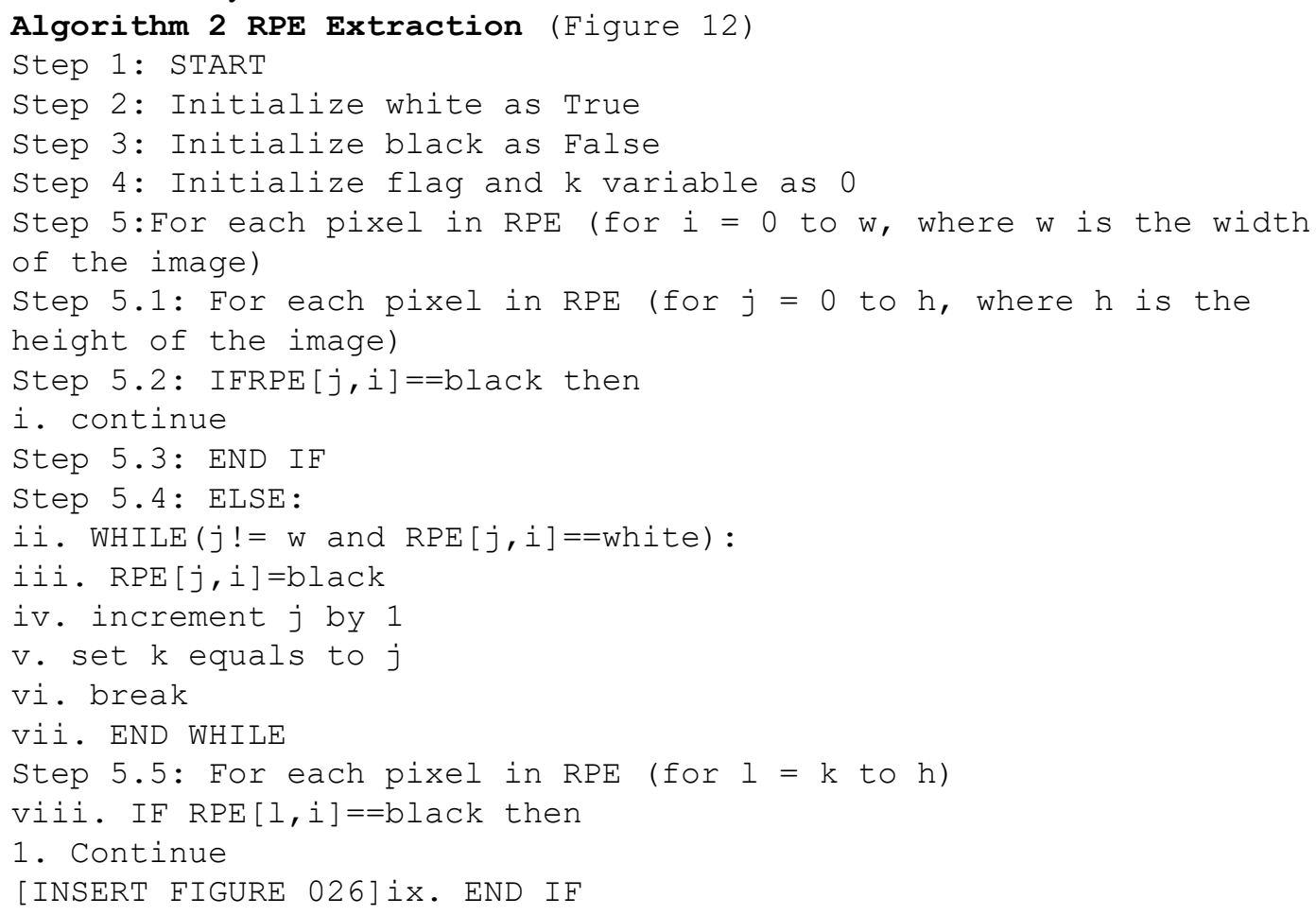




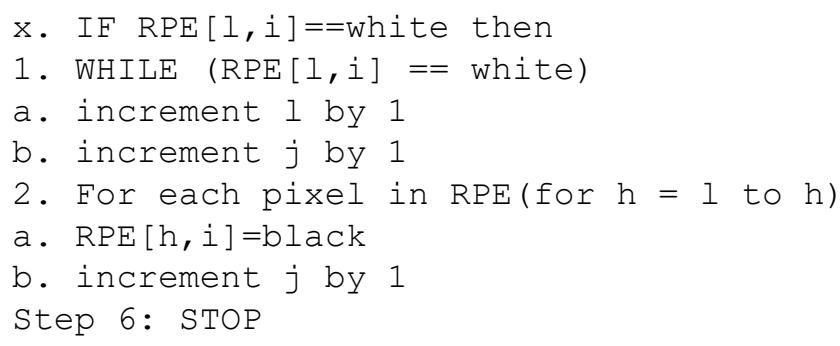

After applying the RPE extraction algorithm, we get the RPE layer, as in Fig. 12. Here below mentioned method is compiled for thickness calculation.

Figure 12. Extracted RPE Layer

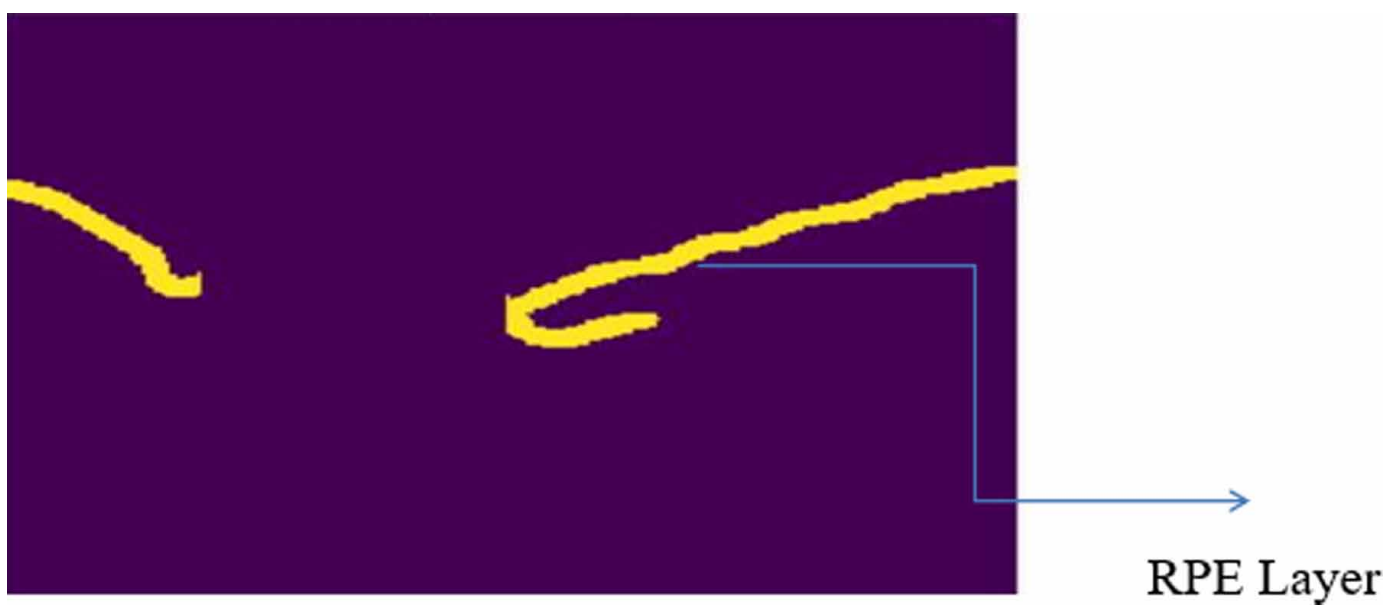

Method for Thickness Calculation

1. Initialize rf variable as 10

2. Initialize $s$ and $m$ variable to 0

3. Initialize white equals to True

4. for $i=0$ to width

a. $s=0$

b. for $j=0$ to height

c. if(binary[j,i] equals white) then

i. increment $s$ by 1

d. $m=m+(r f \star s)$

5. $m=(m+$ ResolutionFactor*s) / (Width*100)

Resolutionfactor $=10$

$\mathrm{s}=$ Number of white pixels in each column

\subsubsection{Cup Diameter and Disc Diameter}

Disc and cup diameter(Koh et al., 2018) (Fig. 13) are used to extract relevant parts of the OCT image and to calculate the Cup to disc ratio. The optic disc is the distance between the endpoints of the RPE layer in the OCT image, and the optic Cup is the total distance between the curves found in the ILM 
Figure 13. Cup Diameter

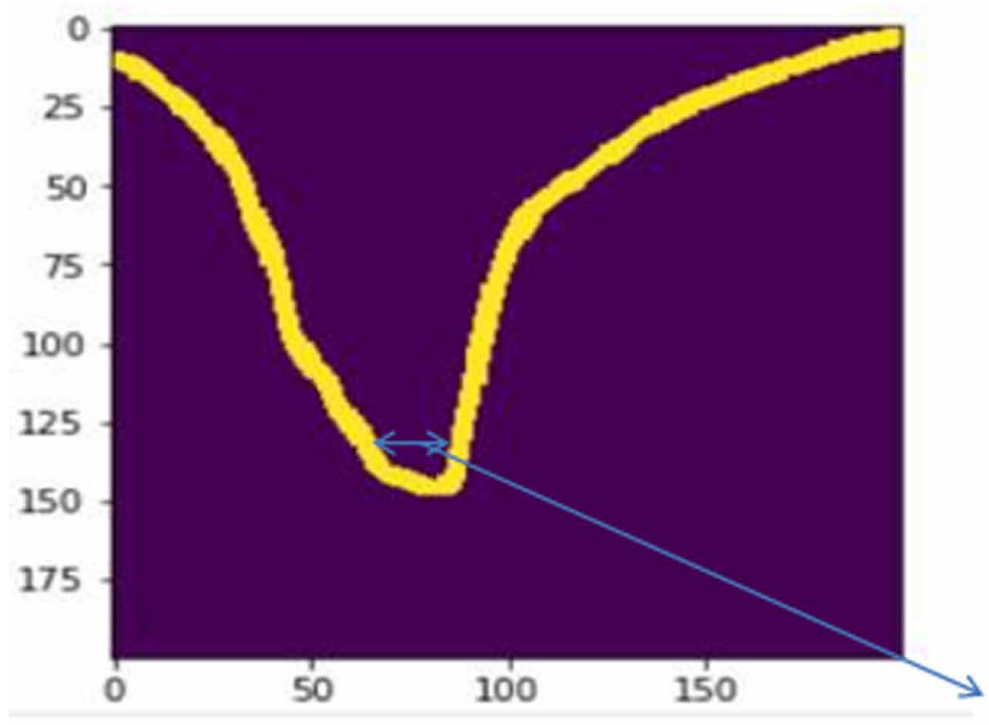

\section{Cup Diameter}

layer. The disc can be a sensitive area. It is also famous as an eyeless spot as the outcome of rods \& cones. Blindspot recognition might be a complicated and hard duty. The disc region is driven in a number of the blood vessels.

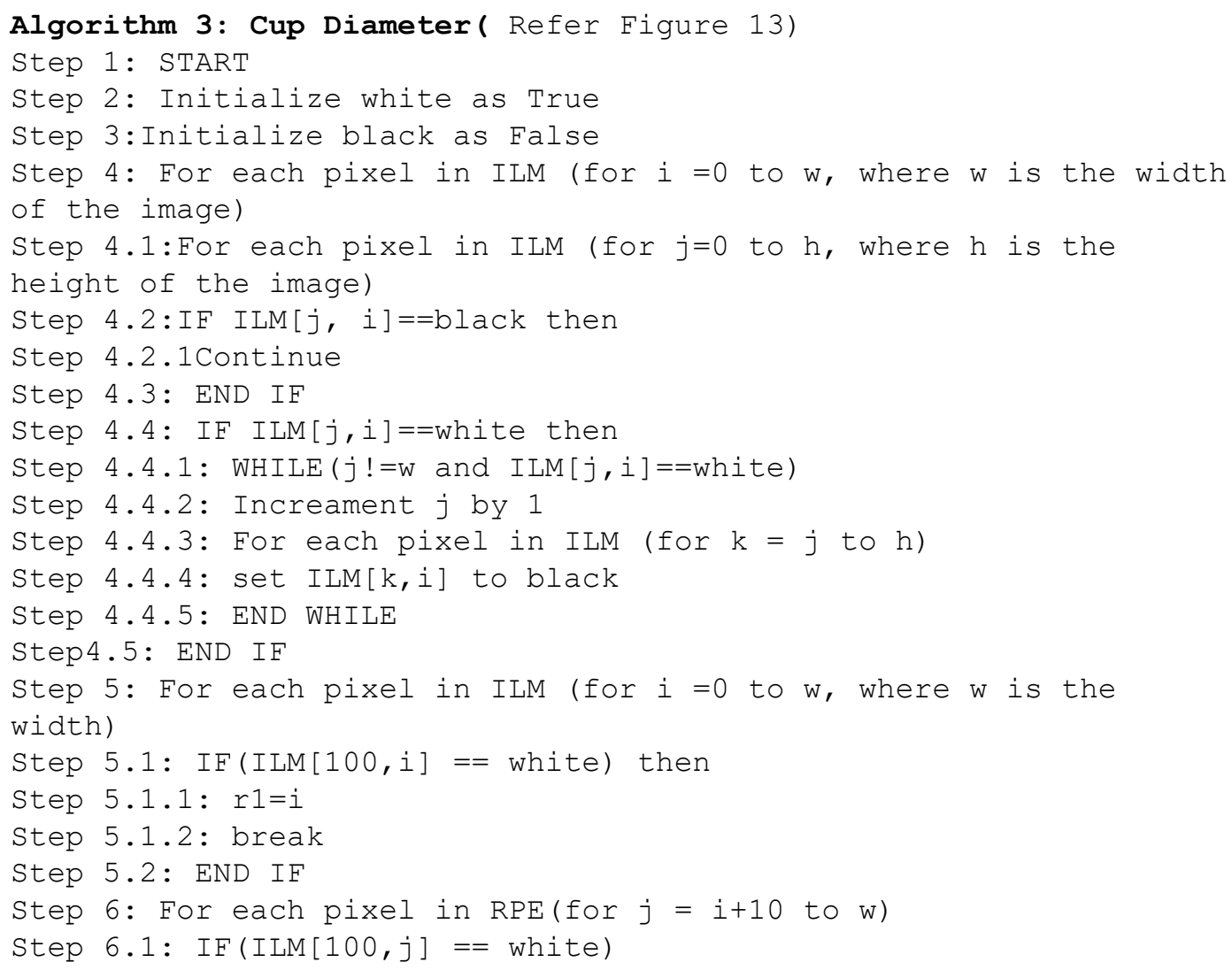


Step 6.1.1: $r 2=j$

Step 6.1.2: break

Step 6.2:END IF

Step 7: Calculate distance between points (r1,100) and (r2,100)

Step 8: STOP

Cup Diameter

\section{Disc Diameter(Algorithm):}

Algorithm 4: Disc Diameter (Refer Fig. 14)

Step 1: START

Step 2: Initialize white as True

Step 3: Initialize black as False

Step 4: Initialize flag and $k$ variable as 0

Step 5:For each pixel in RPE (for $i=0$ to $w$, where $w$ is the width of the image)

Step 5.1: For each pixel in RPE (for $j=0$ to $h$, where $h$ is the height of the image)

Step 5.2: IF RPE[j,i]==black then

i. continue

Step 5.3: END IF

Step 5.4: ELSE:

ii. $\operatorname{WHILE}(j !=w$ and $\operatorname{RPE}[j, i]==$ white $)$ :

iii. $\operatorname{RPE}[j, i]=b l a c k$

iv. increment $j$ by 1

$v$. set $k$ equals to $j$

vi. break

vii. END WHILE

Step 5.5: For each pixel in RPE (for $l=k$ to $h$ )

viii. IF $\operatorname{RPE}[1, i]==b l a c k$ then

3. Continue

ix. END IF

$x$. IF $\operatorname{RPE}[1, i]==$ white then

4. WHILE (RPE $[1, i]==$ white)

a. increment 1 by 1

b. increment $j$ by 1

5. For each pixel in $\operatorname{RPE}$ (for $h=1$ to $h$ )

a. $\operatorname{RPE}[\mathrm{h}, \mathrm{i}]=\mathrm{black}$

b. increment $j$ by 1

Step 6: Initialize r1 as 0

Step 7; Initialize c1 as0

Step 8: For each pixel in RPE (for $i=0$ to $w$, where $w$ is the width)

Step 9: For each pixel in RPE(for $j=0$ to $h$, where $h$ is the

height)

Step 10:IF(image[j][i] == white) then

Step 10.1: set $r 1$ as $j$

Step 10.2: set c1 as i

Step 10.3: break

Step 11: IF ( $j==199)$ :

Step 11.1:break

Step 12 END IF 
Figure 14. Disc Diameter
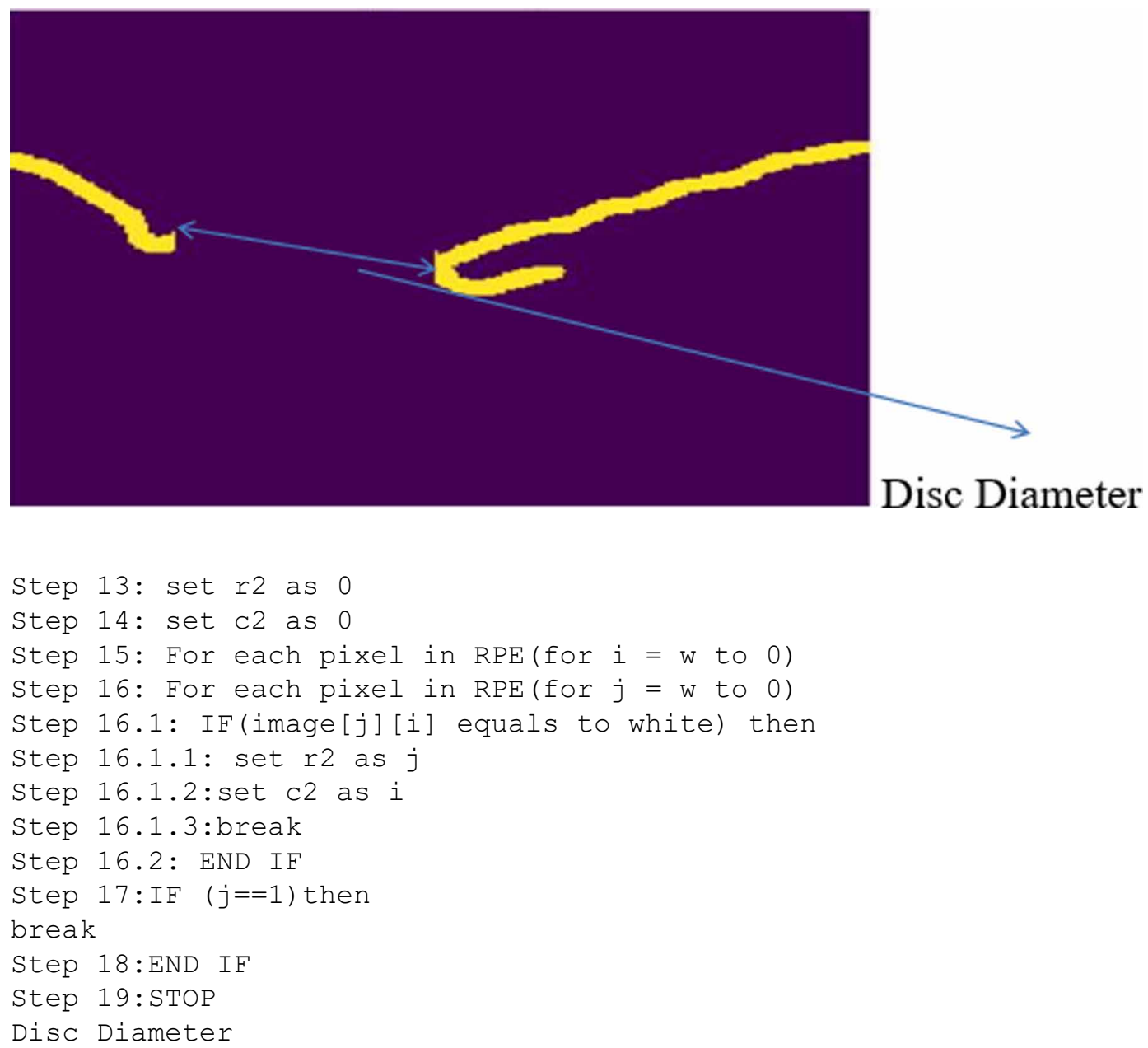

\subsubsection{Cup to Disc Ratio (CDR)}

This is the most significant feature that identifies the presence of Glaucoma. Because of said disease, CDR relation will enlarge that cause's total blindness. Projected CDR endures the detection of this automatic eye disease that empowered ophthalmologists at early identification of glaucoma patients with elevated correctness (Almazroa et al., 2015).

$\mathrm{CDR}=$ Cup diameter/Disc diameter

If $\mathrm{CDR}>=0.5$ subject may be glaucomatous; if $\mathrm{CDR}<0.5$ subject is healthy.

The snapshot of extracted feature values is displayed in Table 2.

\section{RESULTS AND DISCUSSION}

Mendeley Dataset is selected as a subject for the evaluation of the proposed system. Dataset takes both SD-OCT and fundus images however we have worked on OCT only. This experimental study only used SD-OCT images of 70 glaucomatous and 70 healthy patients with resolution $200 * 200$ dpi. 
Table 2. Snapshot of extracted feature values

\begin{tabular}{|c|c|c|c|c|c|}
\hline ILM & RPE & Disc Diameter & Cup Diameter & CDR & Class \\
\hline 136.5615 & 156.8955 & 0.732461603 & 0.31 & 0.423231303 & 1 \\
\hline 162.919 & 98.2355 & 0.763216876 & 0.49 & 0.642016719 & 1 \\
\hline 126.742 & 174.3915 & 0.432897216 & 0.2 & 0.462000462 & 1 \\
\hline 148.5695 & 49.5995 & 0.769415362 & 0.45 & 0.584856125 & 1 \\
\hline 139.144 & 17.745 & 0.6377 & 0.29 & 0.454759291 & 1 \\
\hline 147.34 & 118.29 & 0.655515065 & 0.26 & 0.396631682 & 0 \\
\hline 147.6235 & 146.1175 & 0.71063352 & 0.46 & 0.647312948 & 0 \\
\hline 140.196 & 131.2715 & 0.631585307 & 0.24 & 0.37999335 & 0 \\
\hline 107.384 & 5.3535 & 0.63 & 0.24 & 0.3799 & 0 \\
\hline
\end{tabular}

This work is attempted on the combination of public (MENDELEY) dataset and private dataset of Famous Eye Specialists located nearby the author's hometown. The experimental work is based on $\mathrm{K}$ - folds cross-validation and the entire dataset has been split into 5 -folds because we have chosen the value of $\mathrm{K}$ as 5 .

\subsection{Parameters of performance measurement}

The performance of the proposed methodology is assessed by considering several efficiency measuring metrics such as accuracy, sensitivity, specificity, and precision. The corresponding mathematical equations of these metrics are given below

Sensitivity $=\frac{T P}{(T P+F N)}$

Specificity $=\frac{T N}{(T N+F P)}$

Accuracy $=\frac{(T P+T N)}{(T P+T N+F P+F N)}$

$F 1-$ Score $=2 *\left(\frac{(\operatorname{Pr} \text { ecision } * \text { Re } \text { call })}{(\operatorname{Pr} \text { ecision }+\operatorname{Re} \text { call })}\right)$ 
Precision $=\frac{T P}{(T P+F P)}$

Re call $=\frac{T P}{(T P+F N)}$

Kappa: It is an instrument used for measuring inters and inter- rater reliability for categorical items. It is more robust than simple percent agreement calculation. Kappa statistic is used to control only those instances that may have been correctly classified by chance. This can be calculated using both the observed (total) accuracy and the random accuracy. The maximum value of kappa shows complete agreement.

$p=\frac{(a+d)}{(a+b+c+d)}$

$p s=\left(\frac{(a+b)}{(a+b+c+d)}\right) *\left(\frac{(a+c)}{(a+b+c+d)}\right)$

$p n=\left(\frac{(c+d)}{(a+b+c+d)}\right) *\left(\frac{(b+d)}{(a+b+c+d)}\right)$

$p e=p s+p n$

kappa $=\frac{(p-p e)}{(1-p e)}$

where $\mathrm{a}=$ True Positives, $\mathrm{b}=$ False Positives, $\mathrm{c}=$ False Negatives, $\mathrm{d}=$ True Negatives

\subsection{Combined Models}

Table 3 deliberates the performance analysis of various machine learning classifiers (i.e., KNN, Random forest, LDA, Decision Tree, and SVM) on specificity, sensitivity, accuracy and F1-score.

The above result investigates the performance of various machine learning classifiers. It differentiates among healthy and glaucomatous eyes using OCT parameters. KNN comes out as the best classifier, using 45 parameters, with $97 \%$ accuracy, $100 \%$ sensitivity, and $85.7 \%$ specificity. 
F1-score is 0.92 for glaucomatous and 0.91 for normal follow-through decision tree and for LDA its accuracy is $92 \%$, sensitivity is $100 \%$, specificity is $83.34 \%, \mathrm{~F} 1$ score 0.91 for glaucomatous, 0.92 for normal.

Specificity is concerned with the capability to sense negative results that classify the zeros correctly which is more significant than classifying the ones. Table 4 illustrates the specificity of a variety of the models, i.e., KNN, SVM, Decision Tree, Random Forest, LDA is $85.71 \%, 66 \%, 83.34 \%$, $75 \%$, and $83.34 \%$ respectively.

Table 3. Combined Study of various models

\begin{tabular}{|c|c|c|c|c|c|c|c|c|c|c|}
\hline Sno & Models & Sensitivity & Specificity & \multicolumn{2}{|c|}{ F1-Score } & \multicolumn{2}{|c|}{ Precision } & \multicolumn{2}{|c|}{ Recall } & Accuracy \\
\hline & & & & $\mathbf{0}$ & $\mathbf{1}$ & $\mathbf{0}$ & $\mathbf{1}$ & $\mathbf{0}$ & $\mathbf{1}$ & \\
\hline 1 & KNN & $\mathbf{1 0 0 \%}$ & $\mathbf{8 5 . 7 1 \%}$ & 0.92 & 0.91 & 0.86 & 1.00 & 1.00 & 0.83 & $\mathbf{9 7 \%}$ \\
\hline 2 & SVM & $80 \%$ & $66 \%$ & 0.67 & 0.80 & 0.67 & 0.80 & 0.67 & 0.83 & $75 \%$ \\
\hline 3 & Random Forest & $\mathbf{1 0 0 \%}$ & $75 \%$ & 0.86 & 0.89 & 0.75 & 1.00 & 1.00 & 0.80 & $88 \%$ \\
\hline 4 & LDA & $\mathbf{1 0 0 \%}$ & $83.34 \%$ & 0.91 & 0.92 & 0.83 & 1.00 & 1.00 & 0.86 & $92 \%$ \\
\hline 5 & Decision Tree & $\mathbf{1 0 0} \%$ & $83.34 \%$ & 0.91 & 0.92 & 0.83 & 1.00 & 1.00 & 0.86 & $92 \%$ \\
\hline
\end{tabular}

Sensitivity is the capability to get a positive result. As per Table 4 the sensitivity of a variety of models i.e. KNN, SVM, Decision Tree, Random Forest, LDA are $100 \%, 80 \%, 100 \%, 100 \%, 100 \%$ correspondingly.

Accuracy is the ability to get an accurate classification. The accuracy of various models i.e. KNN, SVM, Decision Tree, Random Forest, LDA are 97\%, 75\%,92\%,88\%,92\% respectively.

F1-Score is the measure of the test's precision. It considers both precision and the remind of the test for computing all scores (Table 3). The F1 Score of a variety of models KNN, SVM, Decision Tree, Random Forest, LDA in the glaucomatous eyes are 0.92, 0.67, 0.91, 0.91, and 0.86 respectively and for the normal eye are $0.91,0.80,0.92,0.89$, and 0.92 respectively.

Correctness is defined as several true positives separated by the number of true positives plus a number of False-Positive(Table 3). The precision of a variety of model that is KNN, SVM, Decision Tree, Random Forest, LDA for Glaucomatous eyes are $0.86,0.67,0.83,0.75,0.83$ respectively and for normal 1.00, 0.80, 1.00, 1.00, 1.00 respectively.

The recall is the capability to find all the related instances in the dataset. The recall of a variety of models i.e. KNN, SVM, Decision Tree, Random Forest, and LDA for Glaucomatous eyes are $1.00,0.67,1.00,1.00$, and 1.00 respectively and for a normal eye is $0.83,0.83,0.86,0.80$, and 0.86 .

Table 4. Kappa and AUC of different models

\begin{tabular}{|c|c|c|c|}
\hline S. No & Models & Kappa & $\begin{array}{c}\text { AUC } \\
\text { (Area Under Curve) }\end{array}$ \\
\hline 1 & KNN & 0.833 & 0.97 \\
\hline 2 & SVM & 0.466 & 0.733 \\
\hline 3 & LDA & 0.833 & 0.929 \\
\hline 4 & Decision Tree & 0.833 & 0.929 \\
\hline 5 & Random Forest & 0.75 & 0.900 \\
\hline
\end{tabular}


After comparing all the selected models (KNN, Decision Tree, SVM, Random Forest and LDA) we get to know that the average accuracy of KNN is $97 \%$, the accuracy of the decision tree, LDA are almost the same i.e. 92\% whereas Random Forest and SVM with $88 \%$ and $75 \%$ respectively.

Kappa: It is used to measure inter and intra rater consistency for categorical items. It is much stronger than the trouble-free percent contract calculation. It measures the agreement of prediction with a true class. -1.0 signifies total agreement if there is no agreement along with the raters other than what could be predictable by chance, Kappa=0(Table 4 and Fig.15).

In this empirical study the highest computed value of kappa is 0.833 for KNN, LDA, and Decision Tree. It represents closeness to perfect agreement means more close to Unity(1) more perfect the agreement will be; it represents rater agree in their classification of an aggregate of closeness to zero $(0)$ indicates that rater had simply guessed every rating.

AUC stands for the Area beneath the ROC curve. It measures the entire two-dimensional areas underneath the entire ROC curve from $(0,0)$ to $(1,1)$. AUC provides an aggregate measure of performance across all possible categorization thresholds. AUC is desirable for two reasons: AUC is a scale-invariant and classification threshold invariant. In our study the highest achieved value of AUC is 0.97 which is in case of KNN (Table 4 and Figure 15).

\subsection{Bar Graph}

In case of bar chart, one axis explain the type of categories while the other will have the numerical values. The bar graph (Fig. 16) represents the accuracy of different classification models.

Here, $\mathrm{X}$-axis of this bar graph represents classification models while Y-axis shows the accuracy of respective models. The height of the bar represents how accurate the classification model is, more the height; better will be the accuracy. Maximum accuracy is achieved by KNN, which is, 97\%(Fig. 16).

\subsection{Receiver Operating Characteristics Curve}

Fig. 17(a)-(e) shows the plot of the ROC curves of different machine learning models. Combined ROC is basically popular in clinical practice which is used to combine the result of two and more diagnostic tests of a classifier to obtain accurate and certain diagnoses. Ideally, a combination of the classifier can be complete by joining the training $\&$ analysis on common datasets with all the classifiers which can be applied; and the standard methods in machine learning (Decision Tree, LDA, etc) can be used for finding an optimized combined categorization system. It is a tradeoff between sensitivity and specificity for every possible cutoff test and the best cutoff has the highest true positive rate whereas on the other hand, having the lowest false positive rate. Fig. 18 presents the ROC and AUC of different classification models in combined fashion.

Figure 15. BarChart representing Kappa and AUC

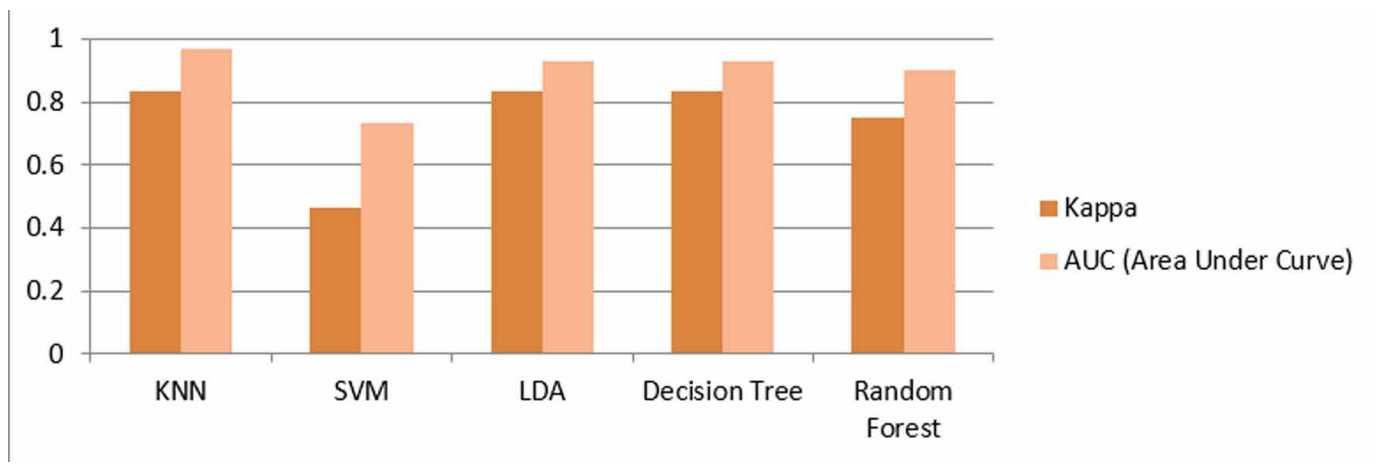


Figure 16. Representation of accuracy

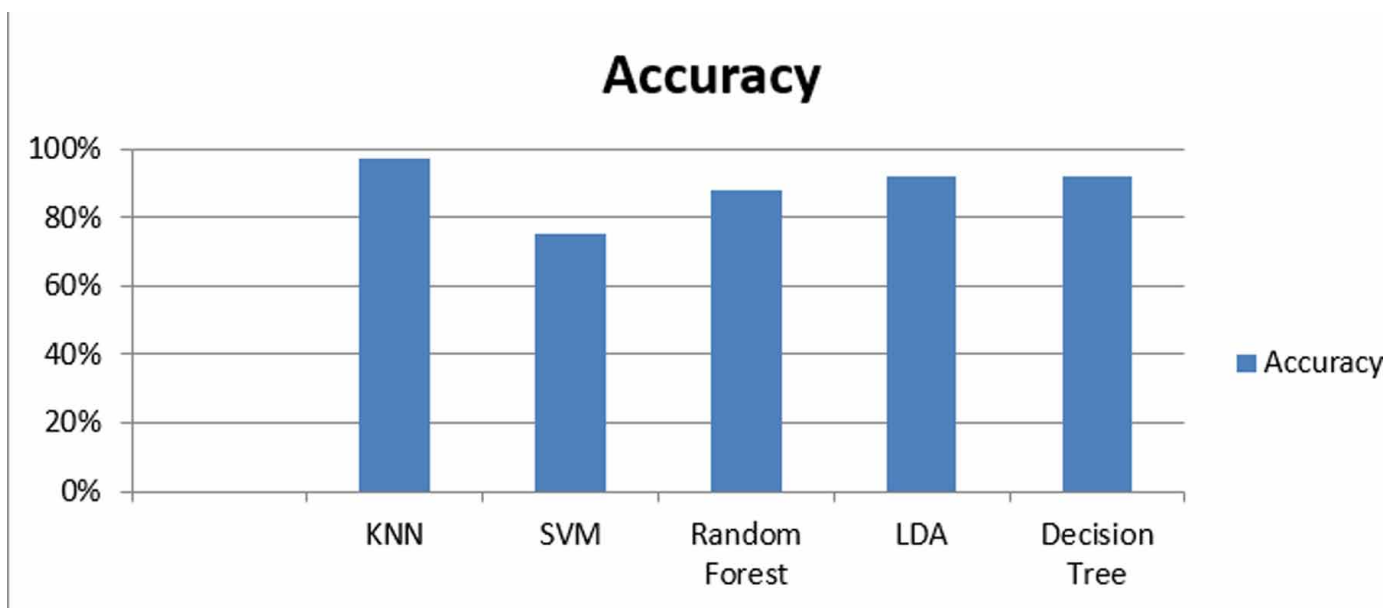

Figure 17. (a) ROC curve of SVM (b) ROC curve of Random Forest (c) ROC curve of LDA (d) ROC curve of Decision Tree (e) ROC curve of KNN

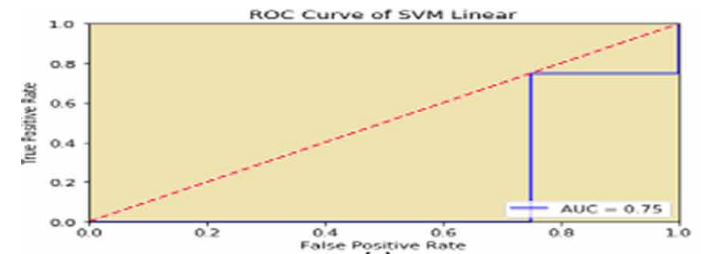

(a)

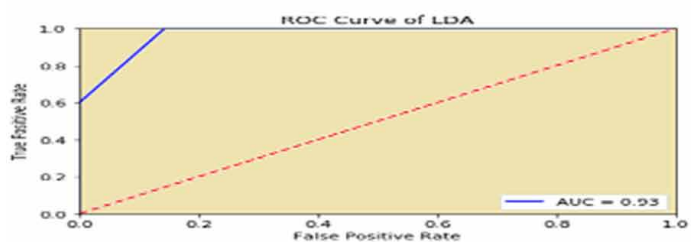

(c)
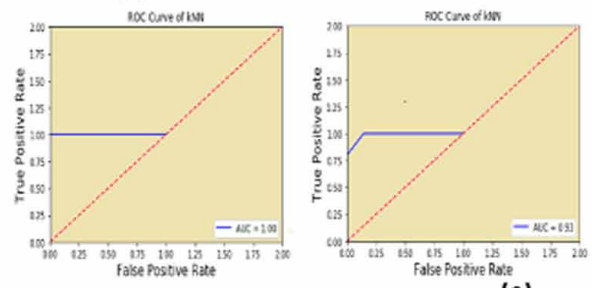

(e)

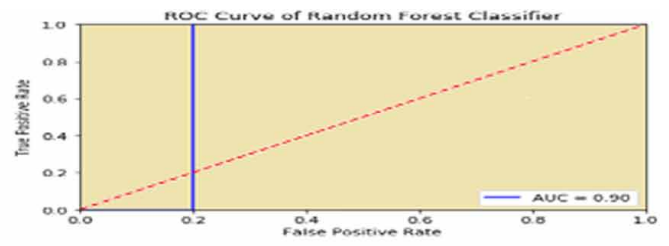

(b)

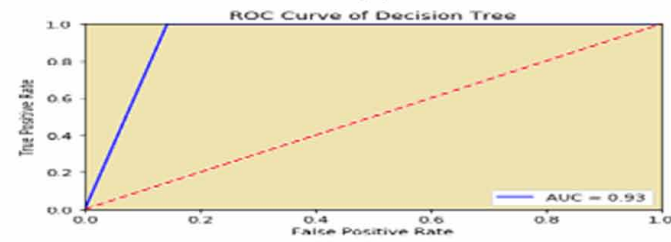

(d)

Running of KNN algorithm for the very first time gives accuracy as $92 \%$ and after that we got $100 \%$. So we took the average accuracy as $97 \%$ and average AUC is also 97\% Fig. 17(e).

Using all 45 features, the largest AROC was achieved with $\mathrm{KNN}(0.93)$ followed by the decision tree (0.93). The sensitivity specificity \& accuracy of KNN, LDA and decision trees are $100 \% 85.71 \%$ $97 \%, 100 \% 83.34 \% 92 \%, 100 \% 83.34 \% 92 \%$ respectively. 


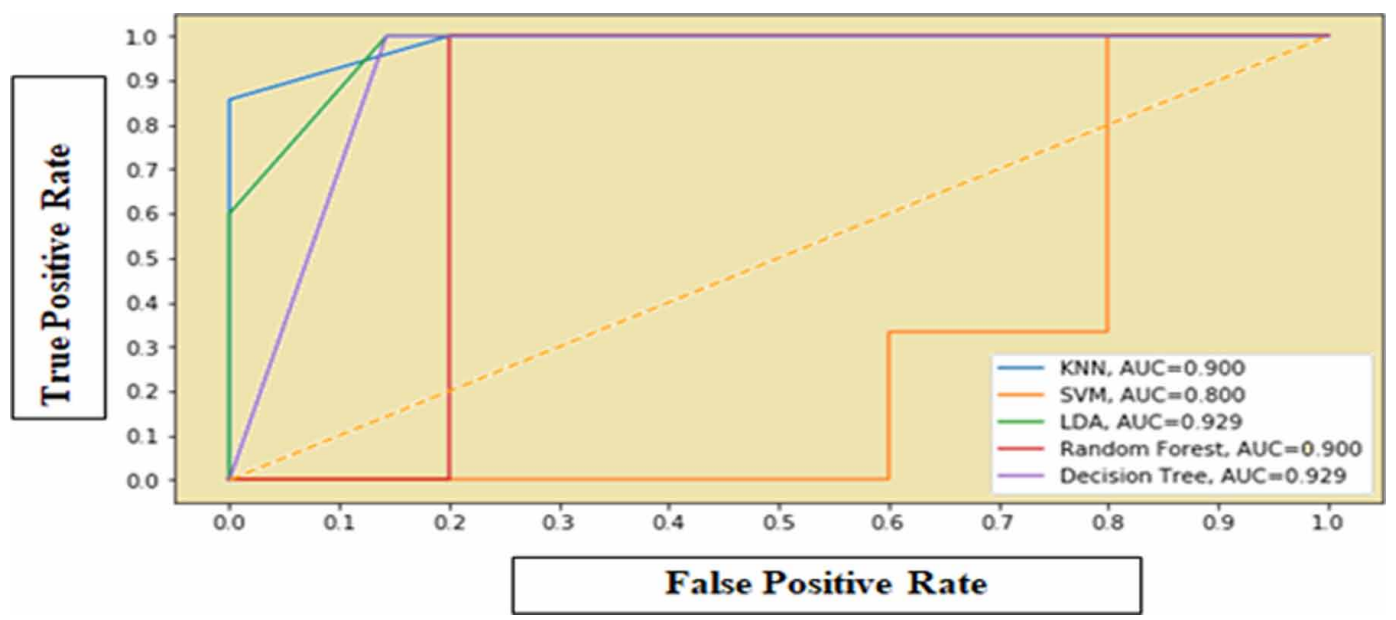

\subsection{Comparison Table}

Table 5 deliberates the performance comparison between four prior published studies with proposed efforts. As a part of in depth investigation, Table 6 is portrayed where comparison is made between some prior published prominent works on fundus images against the proposed work on OCT images as subject of study.

These two comparison tables clearly demonstrate the encouraging performance of the proposed work against the work published on either OCT images or fundus images. Table 6 also proves we have done extensive experimentation to justify the superiority of the best model on the basis of 7 parameters, which is rarely followed by practitioners. Using all 45 features, the largest AROC was achieved with $\mathrm{KNN}(0.97)$ followed by the decision tree $(0.93)$. The sensitivity specificity \& accuracy of KNN, LDA and decision trees are $100 \% 85.71 \%$ 97\%, 100\% 83.34\% 92\%, 100\% 83.34\% 92\% respectively.

\section{CONCLUSION}

The diagnosis of Glaucoma is essential in determining the cause of retinal disease type, which may lead to the damage of the optic nerve those results in progressive and irreversible vision loss. The proposed artificial intelligence based diagnosis model supports the medical practitioners to recognize

Table 5. Comparison table of previously published work versus proposed work

\begin{tabular}{|l|c|c|c|}
\hline \multicolumn{1}{|c|}{ Authors } & Features & Models & Accuracy \\
\hline (Babu et al., 2015) & $\begin{array}{c}\text { CDR, Cup Depth, } \\
\text { Retinal Thickness }\end{array}$ & SVM,BPN & $96.92 \%, 90.76 \%$ \\
\hline (CS, F.,2019) & RNFL & SVM & $92 \%$ \\
\hline (Khalil et al.,2018) & $\begin{array}{l}\text { ILM, Cup Diameter, } \\
\text { Disc Diameter, CDR }\end{array}$ & Decision Tree \\
\hline (An et al.,2019) & Optic Disc, RNFL & Random Forest & $95 \%$ \\
\hline Proposed Methodology & $\begin{array}{c}\text { 40 ORB Features, ILM, } \\
\text { RPE, Cup Diameter, } \\
\text { Disc diameter, CDR }\end{array}$ & $\begin{array}{c}\text { KNN, LDA, Decision Tree, } \\
\text { Random Forest and SVM }\end{array}$ & KNN (97\%) \\
\hline
\end{tabular}


Table 6. Comparison table of previously published work, specifically on fundus images, versus proposed work, on OCT images, (on various efficiency metrics)

\begin{tabular}{|c|c|c|c|c|c|c|c|}
\hline Authors & Sensitivity & Specificity & Precision & Recall & $\begin{array}{c}\text { F1- } \\
\text { Score }\end{array}$ & Kappa & Accuracy \\
\hline $\begin{array}{c}\text { Acharya } \\
\text { et al., 2015 }\end{array}$ & 89.75 & $\mathbf{9 6 . 2}$ & -- & -- & -- & -- & 93.1 \\
\hline $\begin{array}{c}\text { Gajbhiye } \\
\text { et al., 2015 }\end{array}$ & -- & -- & -- & -- & -- & -- & 86.57 \\
\hline $\begin{array}{c}\text { de Souca } \\
\text { et al., 2017 }\end{array}$ & 95.0 & 88.2 & -- & -- & -- & -- & 91.2 \\
\hline $\begin{array}{c}\text { Maheshwari } \\
\text { et al., 2017 }\end{array}$ & -- & -- & -- & -- & -- & -- & 95.19 \\
\hline $\begin{array}{c}\text { Al-Bander } \\
\text { et al., 2017 }\end{array}$ & 85 & 90.8 & -- & -- & -- & -- & 88.2 \\
\hline $\begin{array}{c}\text { Acharya } \\
\text { et al., 2011 }\end{array}$ & -- & -- & -- & -- & -- & -- & 90 \\
\hline $\begin{array}{c}\text { Proposed } \\
\text { (KNN) }\end{array}$ & $\mathbf{1 0 0}$ & 85.71 & $\mathbf{8 6}$ & $\mathbf{1 0 0}$ & $\mathbf{9 2}$ & $\mathbf{8 3}$ & $\mathbf{9 7}$ \\
\hline
\end{tabular}

the presence of Glaucoma with enhanced accuracy. It has been observed that extracted features are interdependent and are combined to build for parametric based investigation for diagnosis of Glaucoma. We have selected and worked on OCT images (healthy eye and glaucomatous eye) due to its numerous advantages over fundus images, meanwhile, it has also been observed that the majority of the researcher community has used fundus images as their subject. We have extracted critical 45 features on which various shortlisted machine learning classifiers have been applied which are-SVM, K-NN, Random Forest, LDA, and Decision tree, for training, testing and finally classification. We have designed self customized algorithms for ILM, RPE, Disc Diameter, CDR, and Cup Diameter feature extraction. Evaluation is performed and analyzed through experimental work. Out of the eight efficiency metrics computed during this work, finding best accuracy is our prime objective. Our proposed method also reaches a sensitivity value to the highest level (100\%) than all other approaches proposed by researchers during recent time. This means that our method is better to detect positive cases, what is the best situation for a medical study. We have also measured the kappa parameter for performance measurement. This study demonstrated that KNN outperformed other state of art techniques in terms of accuracy, sensitivity, and specificity. This study justifies that machine learning systems have an excellent competence to differentiate among healthy \& glaucomatous eyes. This system would help to improve the standard for the glaucoma identification \& improve diagnosis consistency on the basis of OCT derived data. As a future direction, we need to work on monitoring glaucoma progression, its embedding in IoT devices and availability in cloud environments. 


\section{REFERENCES}

Acharya, U. R., Dua, S., Du, X., \& Chua, C. K. (2011). Automated diagnosis of glaucoma using texture and higher order spectra features. IEEE Transactions on Information Technology in Biomedicine, 15(3), 449-455.

Acharya, U. R., Ng, E. Y. K., Eugene, L. W. J., Noronha, K. P., Min, L. C., Nayak, K. P., \& Bhandary, S. V. (2015). Decision support system for the glaucoma using Gabor transformation. Biomedical Signal Processing and Control, 15, 18-26.

Ajesh, F., \& Ravi, R. Hybrid features and optimization-driven recurrent neural network for glaucoma detection. International Journal of Imaging Systems and Technology.

Al-Bander, B., Al-Nuaimy, W., Al-Taee, M. A., \& Zheng, Y. (2017, March). Automated glaucoma diagnosis using a deep learning approach. In 2017 14th International Multi-Conference on Systems, Signals \& Devices (SSD) (pp. 207-210). IEEE.

Almazroa, A., Burman, R., Raahemifar, K., \& Lakshminarayanan, V. (2015). Optic disc and optic cup segmentation methodologies for glaucoma image detection: A survey. Journal of Ophthalmology, 2015.

Amina Jameel, A., \& imran Basit, I. (2018). Detection of Glaucoma using Cup to disc ratio from spectral domain optical coherence tomography images. IEEE Access: Practical Innovations, Open Solutions, 6, 4560-4576.

An, G., Omodaka, K., Hashimoto, K., Tsuda, S., Shiga, Y., Takada, N., \& Nakazawa, T. et al. (2019). Glaucoma diagnosis with machine learning based on optical coherence tomography and color fundus images. Journal of Healthcare Engineering, 2019.

Anushika singh, malay kishore dutta, Khunger, M., Choudhury, T., Satapathy, S. C., \& Ting, K. C. (2019). Automated Detection of Glaucoma Using Image Processing Techniques. In Emerging Technologies in Data Mining and Information Security (pp. 323-335). Springer, Singapore

Anusorn, C. B., Kongprawechnon, W., Kondo, T., Sintuwong, S., \& Tungpimolrut, K. (2013). Image processing techniques for glaucoma detection using the cup-to-disc ratio. Science \& Technology Asia, 22-34.

Babu, T. R., Devi, S., \& Venkatesh, R. (2015). Optic nerve head segmentation using fundus images and optical coherence tomography images for glaucoma detection. Biomedical Papers, 159(4), 607-615.

Bizios, D., Heijl, A., Hougaard, J. L., \& Bengtsson, B. (2010). Machine learning classifiers for glaucoma diagnosis based on classification of retinal nerve fibre layer thickness parameters measured by Stratus OCT. Acta Ophthalmologica, 88(1), 44-52.

Bock, R., Meier, J., Nyúl, L. G., Hornegger, J., \& Michelson, G. (2010). Glaucoma risk index: Automated glaucoma detection from color fundus images. Medical Image Analysis, 14(3), 471-481.

Boulton, M., \& Dayhaw-Barker, P. (2001). The role of the retinal pigment epithelium: Topographical variation and ageing changes. Eye (London, England), 15(3), 384-389.

Burgansky-Eliash, Z., Wollstein, G., Chu, T., Ramsey, J. D., Glymour, C., Noecker, R. J., \& Schuman, J. S. et al. (2005). Optical coherence tomography machine learning classifiers for glaucoma detection: A preliminary study. Investigative Ophthalmology \& Visual Science, 46(11), 4147-4152.

Bussel, I. I., Wollstein, G., \& Schuman, J. S. (2014). OCT for glaucoma diagnosis, screening and detection of glaucoma progression. The British Journal of Ophthalmology, 98(Suppl 2), ii15-ii19.

Chakraborty, C. (2019). Computational approach for chronic wound tissue characterization. Informatics in Medicine Unlocked, 17, 100162.

Chakraborty, C. (2019). Performance analysis of compression techniques for chronic wound image transmission under smartphone-enabled tele-wound network. [IJEHMC]. International Journal of E-Health and Medical Communications, 10(2), 1-20.

CS., F. (2019, August). Glaucoma Detection Using Fundus Images and OCT Images. In Government College of Engineering Kannur, International Conference on Systems, Energy \& Environment (ICSEE). 
de Sousa, J. A., de Paiva, A. C., De Almeida, J. D. S., Silva, A. C., Junior, G. B., \& Gattass, M. (2017). Texture based on geostatistic for glaucoma diagnosis from fundus eye image. Multimedia Tools and Applications, 76(18), 19173-19190.

Deepanshu Tyagi. (2019), "Introduction to ORB (Oriented FAST and Rotated BRIEF)". https://medium.com/ data-breach/introduction-to-orb-oriented-fast-and-rotated-brief-4220e8ec40cf

Dutta, M. K., Issac, A., Minhas, N., \& Sarkar, B. (2016). Image processing based method to assess fish quality and freshness. Journal of Food Engineering, 177, 50-58.

Fatima, M., \& Pasha, M. (2017). Survey of machine learning algorithms for disease diagnostic. Journal of Intelligent Learning Systems and Applications, 9(01), 1.

Gajbhiye, G. O., \& Kamthane, A. N. (2015, December). Automatic classification of glaucomatous images using wavelet and moment feature. In 2015 annual IEEE India conference (INDICON) (pp. 1-5). IEEE.

Gelman, R., Stevenson, W., Prospero Ponce, C., Agarwal, D., \& Christoforidis, J. B. (2015). Retinal damage induced by internal limiting membrane removal. Journal of Ophthalmology, •••, 2015.

Hassan, T., Akram, M. U., Hassan, B., Nasim, A., \& Bazaz, S. A. (2015, September). Review of OCT and fundus images for detection of Macular Edema. In 2015 IEEE International Conference on Imaging Systems and Techniques (IST) (pp. 1-4). IEEE.

Khalil, T., Akram, M. U., Raja, H., Jameel, A., \& Basit, I. (2018). Detection of glaucoma using cup to disc ratio from spectral domain optical coherence tomography images. IEEE Access: Practical Innovations, Open Solutions, 6, 4560-4576.

Khalil, T., Khalid, S., \& Syed, A. M. (2014, August). Review of machine learning techniques for glaucoma detection and prediction. In 2014 Science and Information Conference. IEEE.

Koh, V., Tham, Y. C., Cheung, C. Y., Mani, B., Wong, T. Y., Aung, T., \& Cheng, C. Y. (2018). Diagnostic accuracy of macular ganglion cell-inner plexiform layer thickness for glaucoma detection in a population-based study: Comparison with optic nerve head imaging parameters. PLoS One, 13(6), e0199134.

Lee, W. J., Na, K. I., Ha, A., Kim, Y. K., Jeoung, J. W., \& Park, K. H. (2018). Combined Use of Retinal Nerve Fiber Layer and Ganglion Cell-Inner Plexiform Layer Event-based Progression Analysis. American Journal of Ophthalmology, 196, 65-71.

Maheshwari, S., Pachori, R. B., Kanhangad, V., Bhandary, S. V., \& Acharya, U. R. (2017). Iterative variational mode decomposition based automated detection of glaucoma using fundus images. Computers in Biology and Medicine, 88, 142-149.

Pavithra, G., Manjunath, T. C., \& Lamani, D. (2019, February). Detection of Primary Glaucoma Using ANN with the Help of Back Propagation Algo in Bio-medical Image Processing. In Intelligent Communication Technologies and Virtual Mobile Networks (pp. 48-63). Springer.

Raja, H., Akram, M. U., Khawaja, S. G., Arslan, M., Ramzan, A., \& Nazir, N. (2020). Data on OCT and fundus images for the detection of glaucoma. Data in Brief, $\bullet \bullet \bullet, 105342$.

Rublee, E., Rabaud, V., Konolige, K., \& Bradski, G. (2011). ORB: an efficient alternative to SIFT or SURF. 2011 IE Int Conf on Computer Vision: 2564-2571. Google Scholar Google Scholar Digital Library Digital Library.

Salazar-Gonzalez, A., Kaba, D., Li, Y., \& Liu, X. (2014). Segmentation of the blood vessels and optic disk in retinal images. IEEE Journal of Biomedical and Health Informatics, 18(6), 1874-1886.

Shehryar, T., Akram, M. U., Khalid, S., Nasreen, S., Tariq, A., Perwaiz, A., \& Shaukat, A. (2020). Improved automated detection of glaucoma by correlating fundus and SD-OCT image analysis. International Journal of Imaging Systems and Technology.

Smola, A., \& Vishwanathan, S. V. N. (2008). Introduction to machine learning. Cambridge University, UK, 32(34), 2008.

Wollstein, G., Ishikawa, H., Wang, J., Beaton, S. A., \& Schuman, J. S. (2005). Comparison of three optical coherence tomography scanning areas for detection of glaucomatous damage. American Journal of Ophthalmology, 139(1), 39-43. 
Xu, J., Ishikawa, H., Wollstein, G., \& Schuman, J. S. (2011, August). 3D optical coherence tomography super pixel with machine classifier analysis for glaucoma detection. In 2011 Annual International Conference of the IEEE Engineering in Medicine and Biology Society (pp. 3395-3398). IEEE.

Zilly, J., Buhmann, J. M., \& Mahapatra, D. (2017). Glaucoma detection using entropy sampling and ensemble learning for automatic optic cup and disc segmentation. Computerized Medical Imaging and Graphics, 55, $28-41$.

Law Kumar Singh pursuing his PhD from Sharda University, Greater Noida and completed M. Tech in computer science and engineering from Rajasthan Technical University in 2013 respectively. He is presently serving as Assistant professor in computer science and engineering department, Hindustan College of Science and Engineering, Mathura. He has published many research papers in various international journals and conferences. His research area includes image processing, Machine learning and deep learning.

Munish Khanna is working as an Associate Professor and Head of the computer science and engineering department of Hindustan College of Science and Technology, Mathura,India. He has teaching experience of more than 18 years. He has gained his Ph.D from J.C.Bose University of Science and Technology, YMCA, Faridabad, India. He has completed his MTech from DEI Deemed University, Agra, India and BTech from RGPV Bhopal, India. He has published $02 \mathrm{SCl}$ indexed papers and 10 Scopus indexed papers in internationally recognized reputed journals. 